\title{
A note on traction continuity across an interface in a geometrically non-linear framework
}

2019, Vol. 24(8) 2478-2496

(C) The Author(s) 2018

Article reuse guidelines:

sagepub.com/journals-permissions

DOI: $10.1177 / 1081286518766980$

journals.sagepub.com/home/mms

(9SAGE

\author{
Ali Javili \\ Department of Mechanical Engineering, Bilkent University, Ankara, Turkey
}

Received 2 November 2017; accepted: 5 March 2018

\begin{abstract}
The objective of this contribution is to elaborate on the notion of "traction continuity" across an interface at finite deformations. The term interface corresponds to a zero-thickness model representing the interphase between different constituents in a material. Commonly accepted interface models are the cohesive interface model and the elastic interface model. Both the cohesive and elastic interface models are the limit cases of a generalized interface model. This contribution aims to rigorously analyze the concept of the traction jump for the general interface model. The governing equations of the general interface model in the material as well as spatial configurations are derived and the traction jump across the interface for each configuration is highlighted. It is clearly shown that the elastic interface model undergoes a traction jump in both the material and spatial configurations according to a generalized Young-Laplace equation. For the cohesive interface model, however, while the traction field remains continuous in the material configuration, it can suffer a jump in the spatial configuration. This finding is particularly important since the cohesive interface model is based on the assumption of traction continuity across the interface and that the term "traction" often refers to the spatial configuration and not the material one. Thus, additional care should be taken when formulating an interface model in a geometrically non-linear framework. The theoretical findings for various interface models are carefully illustrated via a series of two-dimensional and three-dimensional numerical examples using the finite element method.
\end{abstract}

\section{Keywords}

General interface model, traction jump, interface elasticity, cohesive interface

\section{Introduction}

Interphases between various constituents in heterogeneous materials can play a crucial role on the overall material response. A common strategy to capture the influence of interphases is to replace the finite thickness interphase by a zero-thickness interface model via various techniques such as computational homogenization of material layers [1,2] or a phase-field approach [3-7]. Owing to the large area-to-volume ratio at small scales, the importance of interfaces grows when reducing the problem dimension. The main objective of this contribution is to elaborate on the notion of traction continuity across an interface.

Interfaces can be categorized into various models according to the continuity of the displacement or traction across the interface. Instead of limiting the discussion here to specific interface models, we start with a general interface model that allows for both the displacement jump as well as the traction jump across the interface. One can readily show that both the cohesive interface model and the elastic interface model are the limit cases of the general interface model. The majority of the contributions on the general interface model deal with small strains and are often understood as asymptotic limits of thin interphases [8-17]. The general interface model here is 
formulated for finite deformations and the implications of this model in a geometrically non-linear framework are discussed. The governing equations in both the material and spatial configuration are derived and the traction jumps thereof associated with each configuration are highlighted.

In passing, it should be mentioned that the elastic interface model is geometrically coherent and the traction jump across the interface results from the stress along the interface following the generalized Young-Laplace equation (see $[18,19]$ among others). The interface elasticity theory [20-25] is a mature field based on the surface elasticity theory of Gurtin and Murdoch [26] and has been further investigated in [27-42] among others. The cohesive interface model accounts for the displacement jump across the interface. The cohesive interface model dates back to the seminal works of Barenblatt $[43,44]$ and Dugdale [45] and has been studied extensively in [46-59] with various applications.

\section{I.I. Organization of the manuscript}

This manuscript is organized as follows. Key contributions of this manuscript, as well as the notation and definitions are introduced next. Section 2 deals with theoretical aspects and the governing equations of continuum mechanics at finite deformations accounting for the general interface model. Basic concepts of the differential geometry required for this manuscript are briefly reviewed in Section 2.1. The kinematics of the problem is explained in Section 2.2. Section 2.3 establishes the balance equations in the material configuration. The governing equations in the spatial configuration are derived in Section 2.4. Section 2.5 deals with the constitutive aspects of material modeling in a thermodynamically consistent framework. Section 3 elucidates the theory via a broad variety of numerical examples using the finite element method. Section 4 concludes this work.

\section{I.2. Key contributions of this manuscript}

The primary objective of this contribution is to investigate on the notion of "traction continuity" across an interface at finite deformations. Using a geometrically exact theory, the governing equations of the general interface model in both the material and spatial configurations are derived and the traction jump across the interface for each configuration is highlighted. The key features and contributions of the current manuscript are as follows:

- to derive the governing equations of the general interface model in both the material and spatial formats;

- $\quad$ to establish the equations entirely based on curvilinear coordinates with no limitations to the dimension;

- $\quad$ to provide the equations for a generic interface with arbitrary geometry and curvature;

- to present two- and three-dimensional numerical examples using finite element modeling (FEM) to illustrate the theory.

\section{I.3. Notation and definitions}

Quantities defined on the interface are distinguished from those in the bulk by a bar placed above the quantity. That is, $\{\bar{\bullet}\}$ refers to an interface variable with its bulk counterpart being $\{\bullet\}$. Throughout the manuscript, surface, interface, and curve quantities are denoted as $\{\hat{\bullet}\},\{\bar{\bullet}\}$, and $\{\tilde{\bullet}\}$, respectively, and therefore distinguished from the bulk quantity $\{\bullet\}$ by an accent on top of the quantity. Direct notation is adopted throughout. Bold letters denote tensors and vectors. The average and jump of a quantity $\{\bullet\}$ over the interface are defined by $\{\{\{\bullet\}\}$ and $\llbracket\{\bullet\} \rrbracket$, respectively, as

$$
\mathbb{L}\{\bullet\} \rrbracket:=\{\bullet\}^{+}-\{\bullet\}^{-}, \quad\left\{\{\{\bullet\}\}:=\frac{1}{2}\left[\{\bullet\}^{+}+\{\bullet\}^{-}\right],\right.
$$

and hold the properties

$$
\llbracket\{\bullet\} \cdot\{\circ\} \rrbracket=\mathbb{L}\{\bullet\} \rrbracket \cdot\left\{\{\{0\}\}+\{\{\{\bullet\}\} \cdot \llbracket\{0\} \rrbracket, \quad\{\{\bullet\} \cdot\{\circ\}\}\}=\left\{\{\{\bullet\}\} \cdot\{\{\{0\}\}\}+\frac{1}{4} \mathbb{L}\{\bullet\} \rrbracket \cdot \llbracket\{0\} \rrbracket .\right.\right.
$$

\section{Theory}

The purpose of this section is to establish the governing equations of continuum mechanics at finite deformations accounting for the general interface model. Detailed expositions on nonlinear continuum mechanics can be found in [60-62] among others. To set the stage, the basic concepts and terminologies corresponding to the fundamentals of differential geometry of two-dimensional manifolds in three-dimensional Euclidean space are 


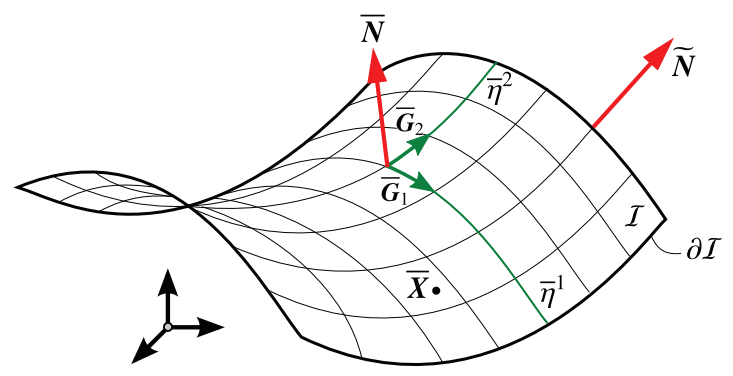

Figure I. The interface as a two-dimensional manifolds in three-dimensional embedding Euclidean space. Coordinates $\overline{\boldsymbol{X}}$ can be parameterized by two coordinates $\bar{\eta}^{1}$ and $\bar{\eta}^{2}$ as $\overline{\boldsymbol{X}}=\overline{\boldsymbol{X}}\left(\bar{\eta}^{1}, \bar{\eta}^{2}\right)$. The covariance interface tangent vectors are denoted by $\overline{\mathbf{G}}_{1}$ and $\overline{\mathbf{G}}_{2}$. The unit normal to the interface is denoted by $\overline{\mathbf{N}}$. The outward unit normal to the boundary of the interface and tangential to the interface is denoted by $\tilde{\mathbf{N}}$.

briefly reviewed next. The content of Section 2.1 is fairly standard and can be found in [63-66], nevertheless, it is required to introduce the notation and to provide a self-contained manuscript. Afterwards, the kinematics of the problem is explained in Section 2.2. Equipped with the required definitions and operators, the balance equations in the material configuration are derived in Section 2.3. The balance equations in the spatial configuration are derived in Section 2.4 and consequences thereof are discussed. Section 2.5 deals with the constitutive behavior of the material for both the bulk and the interface in a thermodynamically consistent manner.

\section{I. Preliminaries}

This section provides a brief introduction to the fundamentals of the differential geometry of two-dimensional manifolds in a three-dimensional embedding Euclidean space. Note that the contents of this section are general and can be applied to both the material and spatial configurations. The use of the capital letters must not imply that the preliminaries hold only in the material configuration.

A two-dimensional (smooth) interface $\mathcal{I}$ in the three-dimensional embedding Euclidean space with coordinates $\overline{\boldsymbol{X}}$ can be parameterized by two coordinates $\bar{\eta}^{\alpha}$ with $\alpha=1,2$ as $\overline{\boldsymbol{X}}=\overline{\boldsymbol{X}}\left(\bar{\eta}^{1}, \bar{\eta}^{2}\right)$. The co-variant (natural) interface basis vectors are $\overline{\boldsymbol{G}}_{\alpha}=\partial_{\bar{\eta}^{\alpha}} \overline{\boldsymbol{X}}$ as shown in Figure 1. The associated contra-variant interface basis vectors $\overline{\boldsymbol{G}}^{\alpha}$ are obtained via $\delta_{\beta}^{\alpha}=\overline{\boldsymbol{G}}^{\alpha} \cdot \overline{\boldsymbol{G}}_{\beta}$ as

$$
\overline{\boldsymbol{G}}_{\alpha}=\bar{G}_{\alpha \beta} \overline{\boldsymbol{G}}^{\beta} \quad \text { with } \bar{G}_{\alpha \beta}=\overline{\boldsymbol{G}}_{\alpha} \cdot \overline{\boldsymbol{G}}_{\beta}=\left[\bar{G}^{\alpha \beta}\right]^{-1} \quad \text { and } \quad \overline{\boldsymbol{G}}^{\alpha}=\bar{G}^{\alpha \beta} \overline{\boldsymbol{G}}_{\beta} \quad \text { with } \bar{G}^{\alpha \beta}=\overline{\boldsymbol{G}}^{\alpha} \cdot \overline{\boldsymbol{G}}^{\beta}=\left[\bar{G}_{\alpha \beta}\right]^{-1} \text {, }
$$

in which $\bar{G}_{\alpha \beta}$ and $\bar{G}^{\alpha \beta}$ are the co-variant and contra-variant interface metric coefficients, respectively. The base vectors $\overline{\boldsymbol{G}}^{3}$ and $\overline{\boldsymbol{G}}_{3}$, normal to $T \mathcal{I}$, are defined by $\overline{\boldsymbol{G}}^{3}:=\overline{\boldsymbol{G}}_{1} \times \overline{\boldsymbol{G}}_{2}$ and $\overline{\boldsymbol{G}}_{3}:=\left[\bar{G}^{33}\right]^{-1} \overline{\boldsymbol{G}}^{3}$ such that $\overline{\boldsymbol{G}}^{3} \cdot \overline{\boldsymbol{G}}_{3}=1$ with the associated metric coefficients $\left[\bar{G}^{33}\right]$ and $\left[\bar{G}_{33}\right]$ following $\left.\left[\bar{G}^{33}\right]=\left|\overline{\boldsymbol{G}}_{1} \times \overline{\boldsymbol{G}}_{2}\right|^{2}=\overline{\operatorname{Det}}_{[} \bar{G}_{\alpha \beta}\right]=\left[\overline{\operatorname{Det}}\left[\bar{G}^{\alpha \beta}\right]\right]^{-1}=$ $\left[\bar{G}_{33}\right]^{-1}$. Consequently, the area element $\mathrm{d} A$ and the unit normal $\overline{\boldsymbol{N}}$ are obtained as $\mathrm{d} A=\left|\overline{\boldsymbol{G}}_{1} \times \overline{\boldsymbol{G}}_{2}\right| \mathrm{d} \bar{\eta}^{1} \mathrm{~d} \bar{\eta}^{2}=$ $\left[\bar{G}^{33}\right]^{1 / 2} \mathrm{~d} \bar{\eta}^{1} \mathrm{~d} \bar{\eta}^{2}$ and $\overline{\boldsymbol{N}}=\left[\bar{G}_{33}\right]^{1 / 2} \overline{\boldsymbol{G}}^{3}=\left[\bar{G}^{33}\right]^{1 / 2} \overline{\boldsymbol{G}}_{3}$, respectively.

Let $\boldsymbol{I}$ denote the ordinary mixed-variant unit tensor of the three-dimensional embedding Euclidian space. The mixed-variant interface unit tensor $\overline{\boldsymbol{I}}$ is defined as

$$
\overline{\boldsymbol{I}}:=\delta_{\beta}^{\alpha} \overline{\boldsymbol{G}}_{\alpha} \otimes \overline{\boldsymbol{G}}^{\beta}=\overline{\boldsymbol{G}}_{\alpha} \otimes \overline{\boldsymbol{G}}^{\alpha}=\boldsymbol{I}-\overline{\boldsymbol{G}}_{3} \otimes \overline{\boldsymbol{G}}^{3}=\boldsymbol{I}-\overline{\boldsymbol{N}} \otimes \overline{\boldsymbol{N}} .
$$

The interface gradient and interface divergence of a vector field $\{\bullet\}$ are defined by

$$
\overline{\operatorname{Grad}}\{\bullet\}:=\partial_{\bar{\eta}^{\alpha}}\{\bullet\} \otimes \overline{\boldsymbol{G}}^{\alpha}=\operatorname{Grad}\{\bullet\} \cdot \overline{\boldsymbol{I}}, \quad \overline{\operatorname{Div}}\{\bullet\}:=\partial_{\bar{\eta}^{\alpha}}\{\bullet\} \cdot \overline{\boldsymbol{G}}^{\alpha}=\operatorname{Grad}\{\bullet\}: \overline{\boldsymbol{I}} .
$$

The curvature tensor $\overline{\boldsymbol{K}}=\bar{K}_{\alpha \beta} \overline{\boldsymbol{G}}^{\alpha} \otimes \overline{\boldsymbol{G}}^{\beta}$ and twice the mean curvature $\bar{K}=\bar{K}_{\alpha}^{\alpha}$ of the interface $\mathcal{I}$ are defined as the negative interface gradient and interface divergence of the interface normal $\bar{N}$, respectively,

$$
\overline{\boldsymbol{K}}:=-\overline{\operatorname{Grad}} \overline{\boldsymbol{N}}=-\partial_{\bar{\eta}^{\beta}} \overline{\boldsymbol{N}} \otimes \overline{\boldsymbol{G}}^{\beta}, \quad \bar{K}:=-\overline{\operatorname{Div}} \overline{\boldsymbol{N}}=-\partial_{\bar{\eta}^{\beta}} \overline{\boldsymbol{N}} \cdot \overline{\boldsymbol{G}}^{\beta} .
$$



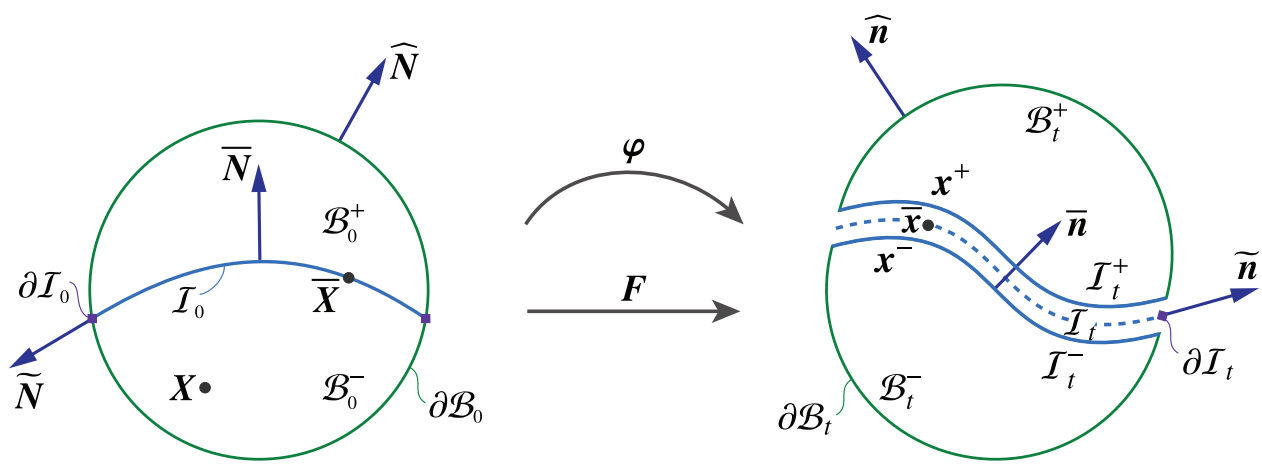

Figure 2. Motion of a continuum body including an interface under finite deformations. The two sides of the body in the material configuration intersect at the interface $\mathcal{I}_{0}$ and, thus, $\llbracket \mathbf{X} \rrbracket=\mathbf{0}$. Through the motion $\varphi$ the interface opens resulting in the displacement jump $\llbracket \mathbf{x} \rrbracket \neq \mathbf{0}$.

Finally, the interface determinant of a second-order tensor field $\{\bullet\}$ is defined by

$$
\overline{\operatorname{Det}}\{\bullet\}:=\frac{\left|\left[\{\bullet\} \cdot \overline{\boldsymbol{G}}_{1}\right] \times\left[\{\bullet\} \cdot \overline{\boldsymbol{G}}_{2}\right]\right|}{\left|\overline{\boldsymbol{G}}_{1} \times \overline{\boldsymbol{G}}_{2}\right|} .
$$

\subsection{Kinematics of the problem}

Let $\mathcal{B}_{0}$ denote the material configuration at time $t=0$ of a continuum body that takes the spatial configuration $\mathcal{B}_{t}$ at any time $t>0$, as shown in Figure 2. The analysis here is quasi-static and the time parameter $t$ is merely a history parameter to order the sequence of events. The interface $\mathcal{I}_{0}$ splits the material configuration $\mathcal{B}_{0}$ into two disjoint subdomains $\mathcal{B}_{0}^{-}$and $\mathcal{B}_{0}^{+}$such that $\mathcal{I}_{0}^{-}:=\mathcal{I}_{0} \cap \partial \mathcal{B}_{0}^{-}$and $\mathcal{I}_{0}^{+}:=\mathcal{I}_{0} \cap \partial \mathcal{B}_{0}^{+}$. The three surfaces $\mathcal{I}_{0}, \mathcal{I}_{0}^{-}$, and $\mathcal{I}_{0}^{+}$coincide in the material configuration, but distance from each other due to the motion $\varphi$ or more precisely, the jump of the motion at the interface $\llbracket \varphi \rrbracket$.

In the material configuration, the placements of the particles in the bulk $\mathcal{B}_{0}$ and on the interface $\mathcal{I}_{0}$ are denoted by $\boldsymbol{X}$ and $\overline{\boldsymbol{X}}$, respectively. The placements $\boldsymbol{X}^{-}$and $\boldsymbol{X}^{+}$on $\mathcal{I}_{0}^{-}$and $\mathcal{I}_{0}^{+}$, respectively, coincide with $\overline{\boldsymbol{X}}$ and $\llbracket X \rrbracket=\mathbf{0}$. The motion $\varphi$ maps the placements of particles from the material configuration to the spatial one via $\boldsymbol{x}=\boldsymbol{\varphi}(\boldsymbol{X})$ where $\boldsymbol{x}$ denotes the placement of particles in the spatial configuration $\mathcal{B}_{t}$. Consequently, $\boldsymbol{X}^{-}$and $\boldsymbol{X}^{+}$ are mapped onto their spatial counterparts $\boldsymbol{x}^{-}$and $\boldsymbol{x}^{+}$on $\mathcal{I}_{t}^{-}$and $\mathcal{I}_{t}^{+}$, respectively, according to $\boldsymbol{x}^{-}=\boldsymbol{\varphi}^{-}(\overline{\boldsymbol{X}})$ and $\boldsymbol{x}^{+}=\varphi^{+}(\bar{X})$. The motion of the interface from the material configuration $\mathcal{I}_{0}$ to the spatial configuration $\mathcal{I}_{t}$ is defined by $\bar{\varphi}$ as $\overline{\boldsymbol{x}}=\bar{\varphi}(\bar{X})$. In the spatial configuration, the two sides of the interface $\mathcal{I}_{t}^{-}$and $\mathcal{I}_{t}^{+}$follow exactly the motion $\bar{\varphi}$ associated with $\mathcal{B}_{t}^{-}$and $\mathcal{B}_{t}^{+}$, respectively. However, this is not the case for the motion $\bar{\varphi}$. The common choice for the motion of the interface $\bar{\varphi}$ is to define the interface $\mathcal{I}_{t}$ as the mid-surface between the two sides $\mathcal{I}_{t}^{-}$and $\mathcal{I}_{t}^{+}$and, hence, $\bar{\varphi}:=\{\{\varphi\}\}$. The jump of the motion across the interface $\llbracket \varphi \rrbracket$ results in the jump $\llbracket \boldsymbol{x} \rrbracket$.

The surface $\mathcal{S}_{0}$ in the material configuration is the boundary $\partial \mathcal{B}_{0}$, but excluding the interface itself as $\mathcal{S}_{0}:=$ $\left[\partial \mathcal{B}_{0}^{-} \cup \partial \mathcal{B}_{0}^{+}\right] \backslash \mathcal{I}_{0}$. The boundary of the interface $\mathcal{I}_{0}$ in the material configuration is the curve $\mathcal{C}_{0}:=\partial \mathcal{I}_{0}$. The bulk, surface, interface, and curve in the spatial configuration are denoted by $\mathcal{B}_{t}, \mathcal{S}_{t}, \mathcal{I}_{t}$, and $\mathcal{C}_{t}$, respectively. The outward unit normal to the surface $\mathcal{S}_{0}$ is denoted by $\widehat{N}$. The unit normal to the interface in the material configuration pointing from the minus side to the plus side of the interface is denoted by $\overline{\boldsymbol{N}}$. The outward unit normal to the boundary of the interface $\mathcal{C}_{0}$ but tangential to the interface $\mathcal{I}_{0}$ is denoted by $\widetilde{N}$. It is crucially important that neither the normal nor the bi-normal to the curve $\mathcal{C}_{0}$ in the sense of the Freńet-Serret formula must necessarily be the same as $\widetilde{N}$. Furthermore, $\widetilde{N}$ is not necessarily normal to the surface $\mathcal{S}_{0}$ as depicted in Figure 2. The unit normals in the spatial configuration are denoted by $\widehat{\boldsymbol{n}}, \overline{\boldsymbol{n}}$, and $\widetilde{\boldsymbol{n}}$ on $\mathcal{S}_{t}, \mathcal{I}_{t}$, and $\mathcal{C}_{t}$, respectively.

The linear deformation maps $\boldsymbol{F}$ and $\overline{\boldsymbol{F}}$ are associated with the non-linear deformation maps $\boldsymbol{\varphi}$ and $\overline{\boldsymbol{\varphi}}$, respectively. The linear deformation maps transform the infinitesimal line elements $\mathrm{d} \boldsymbol{X}$ and $\mathrm{d} \overline{\boldsymbol{X}}$ to their spatial counterparts $\mathrm{d} \boldsymbol{x}$ and $\mathrm{d} \overline{\boldsymbol{x}}$, respectively. The interface deformation gradient $\overline{\boldsymbol{F}}:=\overline{\operatorname{Grad}} \overline{\boldsymbol{\varphi}}$ can be related to the deformation gradient $\boldsymbol{F}:=\operatorname{Grad} \varphi$ via $\overline{\boldsymbol{F}}=\boldsymbol{F} \cdot \overline{\boldsymbol{I}}$ in which $\overline{\boldsymbol{I}}:=\boldsymbol{I}-\overline{\boldsymbol{N}} \otimes \overline{\boldsymbol{N}}$ is the interface identity tensor. The interface deformation gradient $\overline{\boldsymbol{F}}$ is superficial by definition and holds the property $\overline{\boldsymbol{F}} \cdot \overline{\boldsymbol{N}}=\mathbf{0}$. Following the 
standard non-linear continuum mechanics, the important relations on kinematics accounting for interfaces are summarized as

$$
\begin{aligned}
& \boldsymbol{x}=\boldsymbol{\varphi}(\boldsymbol{X}), \quad \mathrm{d} \boldsymbol{x}=\boldsymbol{F} \cdot \mathrm{d} \boldsymbol{X}, \quad \mathrm{d} v=J \mathrm{~d} V \quad, \quad J:=\operatorname{Det} \boldsymbol{F} \quad \text { in } \mathcal{B}_{0}, \\
& \overline{\boldsymbol{x}}=\overline{\boldsymbol{\varphi}}(\overline{\boldsymbol{X}}), \quad \mathrm{d} \overline{\boldsymbol{x}}=\overline{\boldsymbol{F}} \cdot \mathrm{d} \overline{\boldsymbol{X}}, \quad \mathrm{d} a=\bar{J} \mathrm{~d} A \quad, \quad \bar{J}:=\overline{\operatorname{Det}} \overline{\boldsymbol{F}} \quad \text { on } \mathcal{I}_{0},
\end{aligned}
$$

where $\mathrm{d} V$ and $\mathrm{d} v$ denote the volume elements of the bulk in the material and spatial configurations, respectively. Analogously, $\mathrm{d} A$ and $\mathrm{d} a$ denote the area elements of the interface in the material and spatial configurations, respectively. Finally, the Nanson formula on the interface $\mathrm{d} \boldsymbol{a}=J \boldsymbol{F}^{\text {-t }} \cdot \mathrm{d} \boldsymbol{A}$ relates the vectorial area element $\mathrm{d} \boldsymbol{A}:=\mathrm{d} A \overline{\boldsymbol{N}}$ in the material configuration to its spatial counterpart $\mathrm{d} \boldsymbol{a}:=\mathrm{d} a \overline{\boldsymbol{n}}$. The Nanson formula on the surface $\mathcal{S}_{0}$ is formally identical to that on the interface $\mathcal{I}_{0}$, but the area elements are $\mathrm{d} \boldsymbol{A}:=\mathrm{d} A \widehat{\boldsymbol{N}}$ and $\mathrm{d} \boldsymbol{a}:=\mathrm{d} a \widehat{\boldsymbol{n}}$. The line element $\mathrm{d} \boldsymbol{L}$ tangential to the interface and normal to the boundary of the interface in the material configuration maps to its spatial counterpart via the interface normal map $\overline{\operatorname{Cof}} \overline{\boldsymbol{F}}=\bar{J} \overline{\boldsymbol{F}}^{\text {-t }}$ as $\mathrm{d} \boldsymbol{l}=\overline{\operatorname{Cof}} \overline{\boldsymbol{F}} \cdot \mathrm{d} \boldsymbol{L}$ in which $\mathrm{d} \boldsymbol{L}:=\mathrm{d} L \widetilde{\boldsymbol{N}}$ and $\mathrm{d} \boldsymbol{l}:=\mathrm{d} \boldsymbol{l} \widetilde{\boldsymbol{n}}$. Note, the orientations of the line elements $\mathrm{d} \boldsymbol{L}$ and $\mathrm{d} \boldsymbol{l}$ are somewhat counter-intuitive since they are not parallel to the line tangents but to the normals.

\subsection{Balance equations in the material configuration}

The governing equations of the mechanical problem here are the balances of linear and angular momentum, respectively. A commonly accepted strategy to derive the balance equations is via imposing invariance on physically meaningful quantities through an arbitrary rigid-body motion, also attainable from re-parametrization of coordinates. This procedure must be carefully revised to properly incorporate the general interface model. Alternatively, the forthcoming balance equations could be derived in a variational framework [67]. The derivation procedure in the material configuration bears certain similarities to the recent contribution [68]. However, a key feature of this contribution is to cover the governing equations in both the material and spatial configurations. The procedure to derive the balance equations consists of three main steps:

- to establish the integral form of the (external) mechanical power on an arbitrary cutout volume;

- to impose the invariance on the mechanical power with respect to superposed rigid-body motions;

- $\quad$ to localize the integral forms utilizing the arbitrariness of the cutout volume.

In what follows, we adopt exactly the three steps above. In particular, we derive the balance equations in the material configuration first and then derive their spatial format via the Piola identity in the bulk and its extended version on the interface.

First, we write the integral form of the external mechanical power $\mathcal{W}_{0}$ on an arbitrary cutout volume $\mathcal{V}_{0}$ in the material configuration. Throughout this contribution, it is assumed that the surface always remains on the boundary and neither detaches from the bulk nor penetrates the bulk. Thus, the surface is material and the boundaries $\partial \mathcal{V}_{0}^{-}$and $\partial \mathcal{V}_{0}^{+}$assume the same motion as the bulk itself. The same analogy holds for the boundary of the interface namely, the curve $\partial \mathcal{I}_{0}$. Let the force densities in the bulk per unit reference volume, on the surface per unit reference area, on the interface per unit reference area and on the curve per unit reference length be denoted by $\boldsymbol{b}_{0}, \widehat{\boldsymbol{b}}_{0}, \overline{\boldsymbol{b}}_{0}$, and $\widetilde{\boldsymbol{b}}_{0}$, respectively. The external mechanical power in the material configuration $\mathcal{W}_{0}$ reads

$$
\begin{aligned}
\mathcal{W}_{0}=\mathcal{W}_{0}(\dot{\boldsymbol{\varphi}}, \dot{\overline{\boldsymbol{\varphi}}})=\int_{\mathcal{V}_{0}^{-}} \dot{\boldsymbol{\varphi}} \cdot \boldsymbol{b}_{0} \mathrm{~d} V+\int_{\mathcal{V}_{0}^{+}} \dot{\boldsymbol{\varphi}} \cdot \boldsymbol{b}_{0} \mathrm{~d} V+\int_{\partial \mathcal{V}_{0}^{-}} \dot{\boldsymbol{\varphi}} \cdot \widehat{\boldsymbol{b}}_{0} \mathrm{~d} A \\
\\
+\int_{\partial \mathcal{V}_{0}^{+}} \dot{\boldsymbol{\varphi}} \cdot \widehat{\boldsymbol{b}}_{0} \mathrm{~d} A+\int_{\mathcal{I}_{0}} \dot{\overline{\boldsymbol{\varphi}}} \cdot \overline{\boldsymbol{b}}_{0} \mathrm{~d} A+\int_{\partial \mathcal{I}_{0}} \dot{\overline{\boldsymbol{\varphi}}} \cdot \widetilde{\boldsymbol{b}}_{0} \mathrm{~d} L,
\end{aligned}
$$

in which $\dot{\varphi}$ and $\dot{\bar{\varphi}}$ denote the material time derivatives of the bulk and interface motion $\varphi$ and $\bar{\varphi}$, respectively. The surface traction $\widehat{\boldsymbol{b}}_{0}$ can be related to the Piola stress in the material $\boldsymbol{P}$ through the surface normal $\widehat{\boldsymbol{N}}$ via $\widehat{\boldsymbol{b}}_{0}=\boldsymbol{P} \cdot \widehat{\boldsymbol{N}}$ following the Cauchy postulate. For a two-dimensional manifold analogously, the traction $\widetilde{\boldsymbol{b}}_{0}$ on the boundary of the interface $\partial \mathcal{I}_{0}$ is related to the interface stress via $\widetilde{\boldsymbol{b}}_{0}=\overline{\boldsymbol{P}} \cdot \widetilde{\boldsymbol{N}}$ thereby the interface stress $\overline{\boldsymbol{P}}$ is superficial possessing the property $\overline{\boldsymbol{P}} \cdot \overline{\boldsymbol{N}}=\mathbf{0}$. It can be argued [69] that the superficiality property is the 
consequence of a first-order continuum theory. Rewriting the mechanical power (9) in terms of stress instead of traction yields

$$
\begin{aligned}
& \mathcal{W}_{0}=\mathcal{W}_{0}(\dot{\boldsymbol{\varphi}}, \dot{\overline{\boldsymbol{\varphi}}})=\int_{\mathcal{V}_{0}^{-}} \dot{\boldsymbol{\varphi}} \cdot \boldsymbol{b}_{0} \mathrm{~d} V+\int_{\mathcal{V}_{0}^{+}} \dot{\boldsymbol{\varphi}} \cdot \boldsymbol{b}_{0} \mathrm{~d} V+\int_{\partial \mathcal{V}_{0}^{-}} \dot{\boldsymbol{\varphi}} \cdot \boldsymbol{P} \cdot \widehat{\boldsymbol{N}} \mathrm{d} A \\
&+\int_{\partial \mathcal{V}_{0}^{+}} \dot{\boldsymbol{\varphi}} \cdot \boldsymbol{P} \cdot \widehat{\boldsymbol{N}} \mathrm{d} A+\int_{\mathcal{I}_{0}} \dot{\overline{\boldsymbol{\varphi}}} \cdot \overline{\boldsymbol{b}}_{0} \mathrm{~d} A+\int_{\partial \mathcal{I}_{0}} \dot{\overline{\boldsymbol{\varphi}}} \cdot \overline{\boldsymbol{P}} \cdot \tilde{\boldsymbol{N}} \mathrm{d} L .
\end{aligned}
$$

Second, we impose invariance with respect to an arbitrary superposed rigid-body motion on $\mathcal{W}_{0}$. A rigidbody motion is composed of a translation and a rotation. The translational and rotational parts of the motion are captured via the arbitrary (otherwise constant) linear and angular velocities $\boldsymbol{v}$ and $\boldsymbol{\omega}$, respectively, as

$$
\mathcal{W}_{0}=\mathcal{W}_{0}(\dot{\boldsymbol{\varphi}}, \dot{\bar{\varphi}}) \stackrel{!}{=} \mathcal{W}_{0}(\dot{\boldsymbol{\varphi}}+\boldsymbol{v}+\omega \times \boldsymbol{x}, \dot{\bar{\varphi}}+\boldsymbol{v}+\omega \times \overline{\boldsymbol{x}}) \quad \forall \boldsymbol{v}, \boldsymbol{\omega} .
$$

Inserting the invariance condition (11) into (10) renders

$$
\begin{aligned}
& \int_{\mathcal{V}_{0}^{-}} {[v+\omega \times \boldsymbol{x}] \cdot \boldsymbol{b}_{0} \mathrm{~d} V+\int_{\mathcal{V}_{0}^{+}}[\boldsymbol{v}+\boldsymbol{\omega} \times \boldsymbol{x}] \cdot \boldsymbol{b}_{0} \mathrm{~d} V+\int_{\partial \mathcal{V}_{0}^{-}}[\boldsymbol{v}+\boldsymbol{\omega} \times \boldsymbol{x}] \cdot \boldsymbol{P} \cdot \widehat{\boldsymbol{N}} \mathrm{d} A } \\
& \quad+\int_{\partial \mathcal{V}_{0}^{+}}[\boldsymbol{v}+\boldsymbol{\omega} \times \boldsymbol{x}] \cdot \boldsymbol{P} \cdot \widehat{\boldsymbol{N}} \mathrm{d} A+\int_{\mathcal{I}_{0}}[\boldsymbol{v}+\boldsymbol{\omega} \times \overline{\boldsymbol{x}}] \cdot \overline{\boldsymbol{b}}_{0} \mathrm{~d} A+\int_{\partial \mathcal{I}_{0}}[\boldsymbol{v}+\boldsymbol{\omega} \times \overline{\boldsymbol{x}}] \cdot \overline{\boldsymbol{P}} \cdot \tilde{\boldsymbol{N}} \mathrm{d} L=0 \quad \forall \boldsymbol{v}, \boldsymbol{\omega},
\end{aligned}
$$

which, due to arbitrariness of $\boldsymbol{v}$ and $\omega$, could be understood as the integral form of the balance of linear and angular momentum. Since (12) must hold for all arbitrary $\boldsymbol{v}$ and $\boldsymbol{\omega}$, one can set $\boldsymbol{\omega}=\mathbf{0}$ in order to derive the linear momentum balance. Subsequently, the balance of angular momentum in its integral form is obtained by setting $\boldsymbol{v}=\mathbf{0}$.

Finally, through localization of the integral form (12) to an infinitesimal subdomain in the bulk the classical balance of linear and angular momentum for the bulk in the material configuration are obtained as

$$
\operatorname{Div} \boldsymbol{P}+\boldsymbol{b}_{0}=\mathbf{0} \quad \text { and } \quad \boldsymbol{\varepsilon}:\left[\boldsymbol{F} \cdot \boldsymbol{P}^{\mathrm{t}}\right]=\mathbf{0} \Leftrightarrow \boldsymbol{P} \cdot \boldsymbol{F}^{\mathrm{t}}=\boldsymbol{F} \cdot \boldsymbol{P}^{\mathrm{t}},
$$

with $\varepsilon$ being the permutation tensor. Detailed derivation of the localization procedure is omitted here as it is fairly straightforward. In a similar fashion, through localization of (12) to an infinitesimal subdomain on the interface, the balance of linear and angular momentum for the interface in the material configuration are obtained as

$$
\overline{\operatorname{Div}} \overline{\boldsymbol{P}}+\overline{\boldsymbol{b}}_{0}+\llbracket \boldsymbol{t}_{0} \rrbracket=\mathbf{0} \quad \text { and } \quad \boldsymbol{\varepsilon}:\left[\llbracket \varphi \rrbracket \otimes\left\{\left\{\boldsymbol{t}_{0}\right\}\right\}+\overline{\boldsymbol{F}} \cdot \overline{\boldsymbol{P}}^{\mathrm{t}}\right]=\mathbf{0} \quad \text { with } \quad \boldsymbol{t}_{\mathbf{0}}=\boldsymbol{P} \cdot \overline{\boldsymbol{N}} .
$$

It is obvious that in the limit cases of the elastic interface model and the cohesive interface model, the angular momentum balance of the interface simplifies to

$$
\begin{array}{ll}
\text { cohesive interface model: } & \boldsymbol{\varepsilon}:\left[\llbracket \varphi \rrbracket \otimes\left\{\left\{\boldsymbol{t}_{0}\right\}\right]=\mathbf{0} \Leftrightarrow \llbracket \varphi \rrbracket \times\left\{\left\{\boldsymbol{t}_{\mathbf{0}}\right\}\right\}=\left\{\left\{\boldsymbol{t}_{\mathbf{0}}\right\}\right\} \times \llbracket \varphi \rrbracket,\right. \\
\text { elastic interface model: } & \boldsymbol{\varepsilon}:\left[\overline{\boldsymbol{F}} \cdot \overline{\boldsymbol{P}}^{\mathrm{t}}\right]=\mathbf{0} \Leftrightarrow \overline{\boldsymbol{F}} \cdot \overline{\boldsymbol{P}}^{\mathrm{t}}=\overline{\boldsymbol{F}}^{\mathrm{t}} \cdot \overline{\boldsymbol{P}} .
\end{array}
$$

For further details on the balance of angular momentum although only on the cohesive interface model, see e.g. [70].

\subsection{Balance equations in the spatial configuration}

The balance equations (13) and (14) correspond to the balances in the material configuration for the bulk and interface, respectively. The next goal is to establish the balance equations in the spatial configuration and to elaborate on their consequences. In doing so, we start with defining the bulk Cauchy stress $\sigma$ in the spatial configuration. The Cauchy stress can be related to the Piola stress via the equivalence of traction in the material and spatial configurations as

$$
\boldsymbol{P} \cdot \boldsymbol{N} \mathrm{d} A=\boldsymbol{\sigma} \cdot \boldsymbol{n} \mathrm{d} a \quad \Rightarrow \quad \boldsymbol{P}=J \boldsymbol{\sigma} \cdot \boldsymbol{F}^{-\mathrm{t}} \quad \text { or } \quad \boldsymbol{\sigma}=J^{-1} \boldsymbol{P} \cdot \boldsymbol{F}^{\mathrm{t}},
$$


in which the surface Nanson formula $J \boldsymbol{F}^{-\mathrm{t}} \cdot \boldsymbol{N} \mathrm{d} A=\boldsymbol{n} \mathrm{d} a$ plays a key role. Inserting the Cauchy stress $\boldsymbol{\sigma}$ into the balance equations (13), yields

$$
\operatorname{Div}\left(J \boldsymbol{\sigma} \cdot \boldsymbol{F}^{-\mathrm{t}}\right)+\boldsymbol{b}_{0}=\mathbf{0} \quad \text { and } \quad \boldsymbol{\varepsilon}: \boldsymbol{\sigma}^{\mathrm{t}}=\mathbf{0} \Leftrightarrow \boldsymbol{\sigma}=\boldsymbol{\sigma}^{\mathrm{t}},
$$

indicating the familiar symmetry of Cauchy stresses due to the angular momentum balance. The linear momentum balance $(17)_{1}$, can be simplified using the identity

$$
\operatorname{Div}\left(J \boldsymbol{\sigma} \cdot \boldsymbol{F}^{-\mathrm{t}}\right)=\boldsymbol{\sigma} \cdot \operatorname{Div}\left(J \boldsymbol{F}^{-\mathrm{t}}\right)+\operatorname{Grad} \boldsymbol{\sigma}:\left[J \boldsymbol{F}^{-\mathrm{t}}\right]=J \operatorname{Grad} \boldsymbol{\sigma}: \boldsymbol{F}^{-\mathrm{t}}=J \operatorname{div} \boldsymbol{\sigma},
$$

whereby the Piola identity $\operatorname{Div}\left(J \boldsymbol{F}^{\text {-t }}\right)=\mathbf{0}$ is utilized. Eventually, the balances of linear and angular momentum for the bulk in the spatial configuration read

$$
\operatorname{div} \boldsymbol{\sigma}+\boldsymbol{b}_{t}=\mathbf{0} \quad \text { and } \quad \boldsymbol{\sigma}=\boldsymbol{\sigma}^{\mathrm{t}},
$$

in which $\boldsymbol{b}_{t}=\boldsymbol{b}_{0} / J$ is the body force density per unit (current) volume in the spatial configuration.

Similar to the classical Nanson formula that relates the oriented surface elements in the spatial and material configurations, the identity $\bar{J} \overline{\boldsymbol{F}}^{\text {t }} \cdot \widetilde{\boldsymbol{N}} \mathrm{d} L=\widetilde{\boldsymbol{n}} \mathrm{d} l$ can be employed to relate the interface Cauchy stress $\overline{\boldsymbol{\sigma}}$ in the spatial configuration to its material counterpart $\overline{\boldsymbol{P}}$ as

$$
\overline{\boldsymbol{P}} \cdot \tilde{\boldsymbol{N}} \mathrm{d} L=\overline{\boldsymbol{\sigma}} \cdot \widetilde{\boldsymbol{n}} \mathrm{d} l \quad \Rightarrow \quad \overline{\boldsymbol{P}}=\bar{J} \overline{\boldsymbol{\sigma}} \cdot \overline{\boldsymbol{F}}^{\mathrm{t}} \quad \text { or } \quad \overline{\boldsymbol{\sigma}}=\bar{J}^{-1} \overline{\boldsymbol{P}} \cdot \overline{\boldsymbol{F}}^{\mathrm{t}} .
$$

To proceed, an interface identity analogous to the bulk Piola identity is required. The interface Piola identity reads

$$
\overline{\operatorname{Div}}\left(\bar{J} \overline{\boldsymbol{F}}^{\mathrm{t}}\right)=\bar{J} \bar{k} \overline{\boldsymbol{n}},
$$

with $\bar{k}$ indicating twice the mean curvature of the interface in the spatial configuration. To derive the spatial balance equations on the interface, the bulk Cauchy stress $\sigma$ and the interface Cauchy stress $\bar{\sigma}$ are inserted to the interface balance equations (14) resulting in

$$
\operatorname{Div}\left(\bar{J} \overline{\boldsymbol{\sigma}} \cdot \overline{\boldsymbol{F}}^{\mathrm{t}}\right)+\overline{\boldsymbol{b}}_{0}+\llbracket J \boldsymbol{\sigma} \cdot \boldsymbol{F}^{-\mathrm{t}} \rrbracket \cdot \overline{\boldsymbol{N}}=\mathbf{0} \text { and } \boldsymbol{\varepsilon}:\left[\llbracket \varphi \rrbracket \otimes\left\{\left\{\mathbf{J} \boldsymbol{\sigma} \cdot \boldsymbol{F}^{-\mathrm{t}}\right\}\right\} \cdot \overline{\boldsymbol{N}}+\overline{\boldsymbol{F}} \cdot\left[\overline{\mathbf{J}} \overline{\boldsymbol{\sigma}} \cdot \overline{\boldsymbol{F}}^{\mathrm{t}}\right]^{\mathrm{t}}\right]=\mathbf{0},
$$

which, using the relations

$$
\begin{aligned}
& \llbracket J \boldsymbol{\sigma} \cdot \boldsymbol{F}^{-\mathrm{t}} \rrbracket \cdot \overline{\boldsymbol{N}}=\left[J \boldsymbol{\sigma} \cdot \boldsymbol{F}^{-\mathrm{t}} \cdot \boldsymbol{N}\right]^{+}-\left[J \boldsymbol{\sigma} \cdot \boldsymbol{F}^{-\mathrm{t}} \cdot \boldsymbol{N}\right]^{-}=[\widehat{J} \boldsymbol{\sigma} \cdot \boldsymbol{n}]^{+}-[\widehat{J} \boldsymbol{\sigma} \cdot \boldsymbol{n}]^{-}=\llbracket \widehat{J} \boldsymbol{\sigma} \cdot \boldsymbol{n} \rrbracket, \\
& \left.\left\{\left\{J \boldsymbol{\sigma} \cdot \boldsymbol{F}^{-\mathrm{t}}\right\}\right\} \cdot \overline{\boldsymbol{N}}=\frac{1}{2}\left[\left[J \boldsymbol{\sigma} \cdot \boldsymbol{F}^{-\mathrm{t}} \cdot \boldsymbol{N}\right]^{+}+\left[J \boldsymbol{\sigma} \cdot \boldsymbol{F}^{-\mathrm{t}} \cdot \boldsymbol{N}\right]^{-}\right]=\frac{1}{2}[\widehat{J} \boldsymbol{\sigma} \cdot \boldsymbol{n}]^{+}+[\widehat{J} \boldsymbol{\sigma} \cdot \boldsymbol{n}]^{-}\right]=\{\widehat{J} \boldsymbol{\sigma} \cdot \boldsymbol{n}\},,
\end{aligned}
$$

can be simplified to

$$
\left.\operatorname{Div}\left(\bar{J} \overline{\boldsymbol{\sigma}} \cdot \overline{\boldsymbol{F}}^{-\mathrm{t}}\right)+\overline{\boldsymbol{b}}_{0}+\llbracket \widehat{J} \boldsymbol{\sigma} \cdot \boldsymbol{n} \rrbracket=\mathbf{0} \quad \text { and } \quad \boldsymbol{\varepsilon}:[\llbracket \boldsymbol{\varphi} \rrbracket \otimes\{\widehat{J} \boldsymbol{\sigma} \cdot \boldsymbol{n}\}\}+\bar{J} \overline{\boldsymbol{\sigma}}^{\mathrm{t}}\right]=\mathbf{0},
$$

in which $\widehat{J}=\mathrm{d} a / \mathrm{d} A$ is the measure of the area change on each side of the interface. The balance of linear and angular momentum on the interface (24) can be further simplified using the definition of the traction in the spatial configuration $\boldsymbol{t}_{t}=\boldsymbol{\sigma} \cdot \boldsymbol{n}$ and the relation

$$
\begin{aligned}
\operatorname{Div}\left(\bar{J} \overline{\boldsymbol{\sigma}} \cdot \overline{\boldsymbol{F}}^{\text {-t }}\right) & =\bar{\sigma} \cdot \operatorname{Div}\left(\bar{J} \overline{\boldsymbol{F}}^{\text {-t }}\right)+\overline{\operatorname{Grad}} \bar{\sigma}:\left[\bar{J} \overline{\boldsymbol{F}}^{\text {-t }}\right] & & \text { with the interface identity (21) } \\
& =\overline{\boldsymbol{\sigma}} \cdot[\bar{J} \bar{k} \overline{\boldsymbol{n}}]+\bar{J} \overline{\operatorname{div}} \overline{\boldsymbol{\sigma}} & & \text { due to the superficiality property } \\
& =\bar{J} \overline{\operatorname{div}} \overline{\boldsymbol{\sigma}} & &
\end{aligned}
$$

to

$$
\bar{J} \overline{\operatorname{div}} \overline{\boldsymbol{\sigma}}+\overline{\boldsymbol{b}}_{0}+\llbracket \widehat{J} \boldsymbol{t}_{t} \rrbracket=\mathbf{0} \quad \text { and } \quad \boldsymbol{\varepsilon}:\left[\llbracket \varphi \rrbracket \otimes\left\{\left\{\widehat{J} \boldsymbol{t}_{t}\right\}\right\}+\bar{J} \overline{\boldsymbol{\sigma}}^{\mathrm{t}}\right]=\mathbf{0},
$$

alternatively expressed as

$$
\overline{\operatorname{div}} \overline{\boldsymbol{\sigma}}+\overline{\boldsymbol{b}}_{t}+\llbracket \frac{\widehat{J}}{\bar{J}} \boldsymbol{t}_{t} \rrbracket=\mathbf{0} \quad \text { and } \quad \boldsymbol{\varepsilon}:\left[\llbracket \varphi \rrbracket \otimes\left\{\left\{\frac{\widehat{J}}{\bar{J}} \boldsymbol{t}_{t}\right\}\right\}+\overline{\boldsymbol{\sigma}}^{\mathrm{t}}\right]=\mathbf{0} .
$$




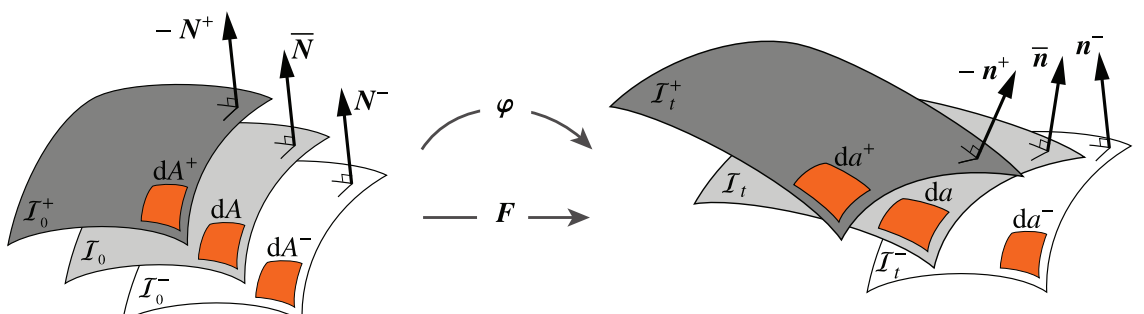

interface in material configuration

interface in spatial configuration

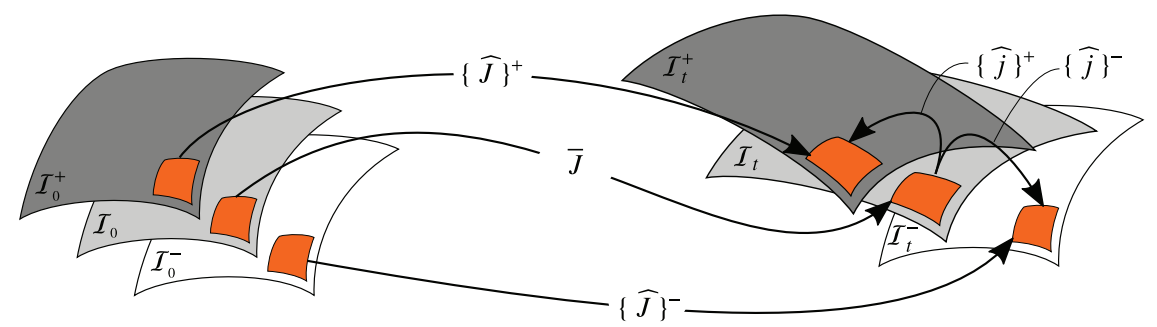

Figure 3. Motion of the general interface under finite deformations. The two sides of the interface are identical in the material configuration but distance from each other in the spatial configuration due to the motion $\varphi$. Unlike the surface Jacobian $\widehat{J}$ that measures the area change from the material to the spatial configuration, the newly defined surface Jacobian $\widehat{j}$ measures the area change of the interface sides with respect to the interface itself within the spatial configuration.

To better understand the balances on the general interface model in the spatial configuration (27), the nonfamiliar ratio $\widehat{J} / \bar{J}$ is further elaborated and its role on the balance equations is explained. The surface Jacobian $\widehat{J}$ on each side of the interface is the measure of the area change; in addition, the interface Jacobian $\bar{J}$ is the measure of the area change of the interface as

$$
\{\widehat{J}\}^{+}=\frac{\mathrm{d} a^{+}}{\mathrm{d} A^{+}}, \quad\{\widehat{J}\}^{-}=\frac{\mathrm{d} a^{-}}{\mathrm{d} A^{-}}, \quad \bar{J}=\frac{\mathrm{d} a}{\mathrm{~d} A} .
$$

Considering that all three area elements $\mathrm{d} A^{+}, \mathrm{d} A^{-}$, and $\mathrm{d} A$ are identical in the material configuration, the ratio $\widehat{J} / \bar{J}$ on each side of the interface reads

$$
\frac{\{\widehat{J}\}^{+}}{\bar{J}}=\frac{\mathrm{d} a^{+}}{\mathrm{d} A^{+}} / \frac{\mathrm{d} a}{\mathrm{~d} A}=\frac{\mathrm{d} a^{+}}{\mathrm{d} a}, \quad \frac{\{\widehat{J}\}^{-}}{\bar{J}}=\frac{\mathrm{d} a^{-}}{\mathrm{d} A^{-}} / \frac{\mathrm{d} a}{\mathrm{~d} A}=\frac{\mathrm{d} a^{-}}{\mathrm{d} a},
$$

which are entirely spatial quantities and can be denoted as

$$
\{\widehat{j}\}^{+}:=\frac{\{\widehat{J}\}^{+}}{\bar{J}}=\frac{\mathrm{d} a^{+}}{\mathrm{d} a}, \quad\{\widehat{j}\}^{-}:=\frac{\{\widehat{J}\}^{-}}{\bar{J}}=\frac{\mathrm{d} a^{-}}{\mathrm{d} a} \quad \Rightarrow \quad \widehat{j}:=\frac{\widehat{J}}{\bar{J}}
$$

The definitions (30) clearly indicate that the non-standard ratio $\widehat{J} / \bar{J}$ in the balance equations of the interface (27) is merely the measure of area change in the spatial configuration. Unlike the surface Jacobian $\widehat{J}$ that measures the area change from the material to the spatial configuration, the newly defined surface Jacobian $\widehat{j}$ measures the area change of the interface sides with respect to the interface itself within the spatial configuration, as illustrated in Figure 3. The balance equations on the general interface model in the spatial configuration (27) can then be expressed as

$$
\left.\overline{\operatorname{div}} \overline{\boldsymbol{\sigma}}+\overline{\boldsymbol{b}}_{t}+\llbracket \widehat{j} \boldsymbol{t}_{t} \rrbracket=\mathbf{0} \quad \text { and } \quad \boldsymbol{\varepsilon}:\left[\llbracket \varphi \rrbracket \otimes\left\{\widehat{j} \boldsymbol{t}_{t}\right\}\right\}+\overline{\boldsymbol{\sigma}}^{\mathrm{t}}\right]=\mathbf{0} .
$$

Table 1 gathers the governing equations of the general interface model in both the material and spatial configurations. The corresponding balance equations in the bulk are included for the sake of completeness and comparison with those associated with the interface. The reduced version of the balance equations for both the 
Table I. Summary of the governing equations of the bulk and interface in the material and spatial configurations. The reduced version of the balance equations for both the elastic interface model and the cohesive interface model are given to better appreciate the differences and similarities among various interface models. The interface force densities $\overline{\boldsymbol{b}}_{0}$ and $\overline{\boldsymbol{b}}_{t}$ are omitted from the simplified interface models for the sake of simplicity.

\begin{tabular}{|c|c|c|}
\hline \multicolumn{3}{|c|}{ Bulk } \\
\hline Material configuration $\mathcal{B}_{0}$ & $\operatorname{Div} \boldsymbol{P}+\boldsymbol{b}_{0}=\mathbf{0}$ & $\varepsilon:\left[\boldsymbol{F} \cdot \boldsymbol{P}^{\mathrm{t}}\right]=\mathbf{0}$ \\
\hline Spatial configuration $\mathcal{B}_{t}$ & $\operatorname{div} \boldsymbol{\sigma}+\boldsymbol{b}_{t}=\mathbf{0}$ & $\boldsymbol{\varepsilon}: \boldsymbol{\sigma}^{\mathrm{t}}=\mathbf{0}$ \\
\hline
\end{tabular}

\begin{tabular}{||l|l|l||}
\hline \multicolumn{2}{||c||}{ General interface model } \\
Material configuration $\mathcal{I}_{0}$ & $\overline{\operatorname{Div}} \overline{\boldsymbol{P}}+\overline{\boldsymbol{b}}_{0}+\llbracket \boldsymbol{t}_{0} \rrbracket=\mathbf{0}$ & $\boldsymbol{\varepsilon}:\left[\llbracket \varphi \rrbracket \otimes\left\{\left\{\boldsymbol{t}_{0}\right\}\right\}+\overline{\boldsymbol{F}} \cdot \overline{\boldsymbol{P}} \mathrm{t}\right]=\mathbf{0}$ \\
Spatial configuration $\mathcal{I}_{t}$ & $\overline{\operatorname{div}} \overline{\boldsymbol{\sigma}}+\overline{\boldsymbol{b}}_{t}+\llbracket \widehat{j} \boldsymbol{t}_{t} \rrbracket=\mathbf{0}$ & $\boldsymbol{\varepsilon}:\left[\llbracket \boldsymbol{\varphi} \rrbracket \otimes\left\{\left\{\widehat{j}_{t}\right\}\right\}+\overline{\boldsymbol{\sigma}}^{\mathrm{t}}\right]=\mathbf{0}$ \\
\hline
\end{tabular}

\begin{tabular}{||c|c||}
\hline \multicolumn{2}{|c||}{ Elastic interface model } \\
\hline$\overline{\operatorname{Div}} \overline{\boldsymbol{P}}+\llbracket \boldsymbol{t}_{0} \rrbracket=\mathbf{0}$ & $\boldsymbol{\varepsilon}:\left[\overline{\boldsymbol{F}} \cdot \overline{\boldsymbol{P}}^{\mathrm{t}}\right]=\mathbf{0}$ \\
$\overline{\operatorname{div}} \overline{\boldsymbol{\sigma}}+\llbracket \boldsymbol{t}_{t} \rrbracket=\mathbf{0}$ & $\boldsymbol{\varepsilon}: \overline{\boldsymbol{\sigma}}^{\mathrm{t}}=\mathbf{0}$ \\
\hline
\end{tabular}

\begin{tabular}{||c|c||}
\hline \multicolumn{2}{||c||}{ Cohesive interface model } \\
$\llbracket \boldsymbol{t}_{0} \rrbracket=\mathbf{0}$ & $\boldsymbol{\varepsilon}:\left[\llbracket \varphi \rrbracket \otimes\left\{\left\{\boldsymbol{t}_{0}\right\}\right]=\mathbf{0}\right.$ \\
$\llbracket \widehat{j}_{t} \rrbracket=\mathbf{0}$ & $\boldsymbol{\varepsilon}:$ \\
& {$\left[\llbracket \varphi \rrbracket \otimes\left\{\hat{j} \boldsymbol{t}_{t}\right\}\right]=\mathbf{0}$} \\
\hline
\end{tabular}

elastic interface model and the cohesive interface model are given to better appreciate the differences and similarities among various interface models. The interface force densities $\overline{\boldsymbol{b}}_{0}$ and $\overline{\boldsymbol{b}}_{t}$ are omitted from the simplified interface models for the sake of simplicity. Nonetheless, it seems rather impossible to apply an external force density to the cohesive interface model due to the lack of its own thermodynamic structure.

The primary objective of this contribution is to investigate on the notion of the traction jump across an interface in both the material and spatial configurations. To do so, the linear balance equation across the cohesive interface model in the spatial configuration shall be further expanded as

$$
\left.\llbracket \widehat{j} \boldsymbol{t}_{t} \rrbracket=\mathbf{0} \quad \Rightarrow \quad \llbracket \widehat{\mathbf{j}} \rrbracket\left\{\boldsymbol{t}_{\mathbf{t}}\right\}\right\}+\left\{\{\hat{\mathbf{j}}\} \llbracket \llbracket \boldsymbol{t}_{\mathbf{t}} \rrbracket=\mathbf{0} \quad \Rightarrow \quad\left\{\{\hat{\boldsymbol{j}}\} \llbracket \llbracket \boldsymbol{t}_{\mathbf{t}} \rrbracket=-\llbracket \widehat{\boldsymbol{j}} \rrbracket\left\{\boldsymbol{t}_{\mathbf{t}}\right\}\right] \quad \Rightarrow \quad \llbracket \boldsymbol{t}_{\mathbf{t}} \rrbracket=-\frac{\llbracket \widehat{\boldsymbol{j}} \rrbracket}{\{\widehat{\boldsymbol{j}}\}\}}\left\{\left\{\boldsymbol{t}_{\mathbf{t}}\right\},\right.\right.
$$

indicating that the traction jump across the cohesive interface in the spatial configuration is obviously nonvanishing, in general. Note, the term $\llbracket \widehat{j} \rrbracket$ indicates the area change across the interface in the spatial configuration is non-zero in general. Furthermore, the average traction $\left\{\left\{\boldsymbol{t}_{t}\right\}\right\}$ is exactly the traction between the two sides of the cohesive interface and, hence, not vanishing as long as there exists a bonding force across the interface. In the context of the cohesive interface model, the interface traction is related to the displacement jump across the interface via a traction-separation law that describes the constitutive behavior of the interface. Finally, Table 2 collects the traction jumps for both the elastic and cohesive interface models in the material as well as the spatial configuration. It is clear that the traction jump across the elastic interface model does not vanish in general and is present in both configurations. However, for the cohesive interface model the traction jump vanishes only in the material configuration but not in the spatial configuration. This finding is extremely important when formulating the cohesive interface model at finite deformations since often the traction continuity condition is assumed for the cohesive interface model without specifically mentioning whether it is Piola or Cauchy traction. This could be particularly misleading since, without an explicit indication, the notion of traction usually refers to the Cauchy traction corresponding to the spatial configuration and not the material one. 
Table 2. Traction jumps for both the elastic and cohesive interface models in the material as well as the spatial configuration.

\begin{tabular}{||l|c|c||}
\hline & Material configuration $\mathcal{I}_{0}$ & Spatial configuration $\mathcal{I}_{t}$ \\
\hline Elastic interface & $\llbracket \boldsymbol{t}_{0} \rrbracket=-\overline{\mathrm{Div}} \overline{\boldsymbol{P}} \neq \mathbf{0}$ & $\llbracket \boldsymbol{t}_{t} \rrbracket=-\overline{\operatorname{div}} \overline{\boldsymbol{\sigma}} \neq \mathbf{0}$ \\
Cohesive interface & $\llbracket \boldsymbol{t}_{0} \rrbracket=\mathbf{0}$ & $\left.\llbracket \boldsymbol{t}_{t} \rrbracket=-\frac{\llbracket \widehat{j} \rrbracket}{\left\{\left\{\widehat{j}_{j}\right\}\right.}\left\{\boldsymbol{t}_{t}\right\}\right\} \neq \mathbf{0}$ \\
\hline
\end{tabular}

\subsection{Constitutive material behavior}

The governing equations of the interface are essentially derived via imposing invariance on the mechanical power with respect to arbitrary superposed rigid-body motions. Next, the mechanical power due to external force fields is viewed as the external power and is incorporated into a thermodynamically consistent framework. In doing so, the constitutive behavior of the bulk and interface must follow from the Coleman-Noll procedure. The external power densities in the bulk and on the interface in the material configuration, respectively, read

$$
\mathscr{W}_{0}=\boldsymbol{P}: \dot{\boldsymbol{F}} \quad \text { and } \quad \overline{\mathscr{W}}_{0}=\overline{\boldsymbol{P}}: \dot{\overline{\boldsymbol{F}}}+\left\{\left\{\boldsymbol{t}_{0}\right\}\right\} \cdot \llbracket \dot{\boldsymbol{\varphi}} \rrbracket
$$

The derivations to obtain the external power densities $\mathscr{W}_{0}$ and $\overline{\mathscr{W}}_{0}$ from the global expression (10) are omitted for the sake of brevity.

To proceed, the free energy densities in the bulk $\psi_{0}$ and on the interface $\bar{\psi}_{0}$ are introduced as Legendre transformations of their corresponding internal energies. The bulk free energy density $\psi_{0}$ is defined per unit reference volume and similarly, the interface free energy density $\bar{\psi}_{0}$ is defined per unit reference area and both are associated with the material configuration. From the definitions of the free energies, the Clausius-Duhem dissipation inequalities in the bulk and on the interface in the material configuration read

$$
\mathscr{D}_{0}=\mathscr{W}_{0}-\dot{\psi}_{0} \geq 0 \quad \text { and } \quad \overline{\mathscr{D}}_{0}=\overline{\mathscr{W}}_{0}-\dot{\bar{\psi}}_{0} \geq 0
$$

To further exploit the dissipation inequalities (34), we limit the discussion here to (reversible) hyperelastic models in the bulk and on the interface. Inspired by the working densities (33), the bulk free energy density is assumed to be a function of the deformation gradient $\boldsymbol{F}$. Similarly, we allow the interface free energy to depend on the interface deformation gradient $\overline{\boldsymbol{F}}$ and the displacement jump across the interface $\llbracket \varphi \rrbracket$. The free energy densities in the bulk and on the interface thus read

$$
\psi_{0}=\psi_{0}(\boldsymbol{F}) \quad \text { and } \quad \bar{\psi}_{0}=\bar{\psi}_{0}(\overline{\boldsymbol{F}}, \llbracket \boldsymbol{\varphi} \rrbracket)
$$

Inserting working densities (33) and free energy densities (35) into the dissipation inequalities (34) yields

$$
\begin{array}{ll}
\mathscr{D}_{0}=\boldsymbol{P}: \dot{\boldsymbol{F}}-\frac{\partial \psi_{0}}{\partial \boldsymbol{F}}: \dot{\boldsymbol{F}} \geq 0 & \text { in } \mathcal{B}_{0}, \\
\overline{\mathscr{D}}_{0}=\overline{\boldsymbol{P}}: \dot{\overline{\boldsymbol{F}}}+\left\{\left\{\boldsymbol{t}_{0}\right\}\right\} \cdot \llbracket \dot{\boldsymbol{\varphi}} \rrbracket-\frac{\partial \bar{\psi}_{0}}{\partial \overline{\boldsymbol{F}}}: \dot{\overline{\boldsymbol{F}}}-\frac{\partial \bar{\psi}_{0}}{\partial \llbracket \boldsymbol{\varphi} \rrbracket} \cdot \llbracket \dot{\boldsymbol{\varphi}} \rrbracket \geq 0 & \text { on } \mathcal{I}_{0} .
\end{array}
$$

Reordering the terms to better see the structure of the dissipation inequalities furnishes

$$
\begin{aligned}
\mathscr{D}_{0}=\left[\boldsymbol{P}-\frac{\partial \psi_{0}}{\partial \boldsymbol{F}}\right]: \dot{\boldsymbol{F}} \geq 0 & \text { in } \mathcal{B}_{0}, \\
\overline{\mathscr{D}}_{0}=\left[\overline{\boldsymbol{P}}-\frac{\partial \bar{\psi}_{0}}{\partial \overline{\boldsymbol{F}}}\right]: \dot{\boldsymbol{F}}+\left[\left\{\left\{\boldsymbol{t}_{0}\right\}-\frac{\partial \bar{\psi}_{0}}{\partial \llbracket \boldsymbol{\varphi} \rrbracket}\right] \cdot \llbracket \dot{\boldsymbol{\varphi}} \rrbracket \geq 0\right. & \text { on } \mathcal{I}_{0} .
\end{aligned}
$$


To extract thermodynamically consistent constitutive laws within the context of equilibrium thermodynamics, the dissipation inequalities (37) can be sufficiently satisfied through the relations

$$
\begin{array}{lll}
\boldsymbol{P}=\frac{\partial \psi_{0}}{\partial \boldsymbol{F}} & \text { in } \mathcal{B}_{0}, \\
\overline{\boldsymbol{P}}=\frac{\partial \bar{\psi}_{0}}{\partial \overline{\boldsymbol{F}}}, \quad\left\{\left\{\boldsymbol{t}_{0}\right\}\right\}=\frac{\partial \bar{\psi}_{0}}{\partial \llbracket \boldsymbol{\varphi} \rrbracket} & \text { on } \mathcal{I}_{0},
\end{array}
$$

which are indeed the constitutive laws for $\boldsymbol{P}, \overline{\boldsymbol{P}}$ and $\left\{\left\{\boldsymbol{t}_{0}\right\}\right\}$, respectively. Note that for a cohesive interface model, $\left\{\left\{\boldsymbol{t}_{0}\right\}\right\}$ is indeed related to the displacement jump $\llbracket \varphi \rrbracket$ via a traction-separation law. More precisely and somewhat surprisingly, the constitutive law for the interface (38) reveals that a thermodynamically consistent tractionseparation law relates the Piola traction in the material configuration to the displacement jump in the spatial configuration.

The constitutive relations (38) clearly show that free energy densities $\psi_{0}$ and $\bar{\psi}_{0}$ in the bulk and on the interface, respectively, must be specified to describe the material behavior. For the material response of the bulk, a hyperelastic neo-Hookean free energy density in the material configuration

$$
\psi_{0}(\boldsymbol{F})=\frac{1}{2} \mu[\boldsymbol{F}: \boldsymbol{F}-3-2 \ln J]+\frac{1}{2} \lambda\left[\frac{1}{2}\left[J^{2}-1\right]-\ln J\right] \quad \text { with } \quad J=\operatorname{Det} \boldsymbol{F},
$$

is chosen with $\lambda$ and $\mu$ being the first and second Lamé constants, respectively. This choice for the free energy density is suitable for rubber-like materials and benefits from the original ideas of Ogden [71] to capture the macroscopic behavior of nearly incompressible soft polymers. Alternatively, it is possible to develop physically interpretable and micro-mechanically motivated material models as discussed in [72] among others. For the interface, the material response is additively decomposed into a tangential part along the interface and an orthogonal part across the interface as

$$
\bar{\psi}_{0}(\overline{\boldsymbol{F}}, \llbracket \varphi \rrbracket)=\bar{\psi}_{0}^{\|}(\overline{\boldsymbol{F}})+\bar{\psi}_{0}^{\perp}(\llbracket \varphi \rrbracket) .
$$

Note that this decomposition is imposed only for the sake of simplicity and to better distinguish the tangential (in-plane) resistance from the orthogonal one. In addition, this decomposition shows more clearly how both the elastic interface model and cohesive interface model can be retrieved from the general form. In analogy to the bulk, the elastic resistance along the interface follows from a hyperelastic neo-Hookean free energy density. For the orthogonal behavior of the interface, the commonly accepted and intuitive cohesive response with quadratic energy in terms of the displacement jump across the interface is assumed. The free energy density of the interface in the material configuration is chosen as

$$
\bar{\psi}_{0}(\overline{\boldsymbol{F}}, \llbracket \varphi \rrbracket)=\frac{1}{2} \bar{\mu}[\overline{\boldsymbol{F}}: \overline{\boldsymbol{F}}-2-2 \ln \bar{J}]+\frac{1}{2} \bar{\lambda}\left[\frac{1}{2}\left[\bar{J}^{2}-1\right]-\ln \bar{J}\right]+\frac{1}{2} \bar{\alpha} \llbracket \varphi \rrbracket^{2} \quad \text { with } \quad \bar{J}=\overline{\operatorname{Det}} \overline{\boldsymbol{F}},
$$

in which $\bar{\mu}, \bar{\lambda}$, and $\bar{\alpha}$ are the interface material parameters. In a three-dimensional setting, the interface is a twodimensional manifold and in a two-dimensional setting a one-dimensional manifold or essentially a curve. For a two-dimensional setting, it is redundant to introduce two material parameters for the in-plane elastic response of the interface and hence, either $\bar{\mu}$ or $\bar{\lambda}$ could vanish, without loss of generality.

\section{Illustration of the theory}

The main goal of this section is to elucidate the theory and, in particular, the traction jump across an interface via numerical examples. In particular, the Piola traction jump $\llbracket \boldsymbol{t}_{0} \rrbracket$ is compared against the Cauchy traction jump $\llbracket \boldsymbol{t}_{t} \rrbracket$. The two-dimensional plane-strain numerical examples correspond to the deformation of the unit square shown in Figure 4. The domain is partitioned into two disjoint subdomains $\mathcal{B}_{0}^{-}$and $\mathcal{B}_{0}^{+}$by the interface $\mathcal{I}_{0}$. The specimen is stretched by applying prescribed displacements on its edges. Lateral deformations are prevented and, hence, the width of the specimen cannot change. The two-dimensional example is devised such that it clearly demonstrates the interface behavior due to the resistance along the interface or orthogonal to the interface. For the given boundary conditions, a straight interface could not properly capture the complete interface behavior and, therefore, the interface here is assumed to be curved.

The material parameters are chosen to amplify the impact of the interface such that the influences of different interface models are distinctly noticeable. The purpose of the examples here is to better understand the notion 

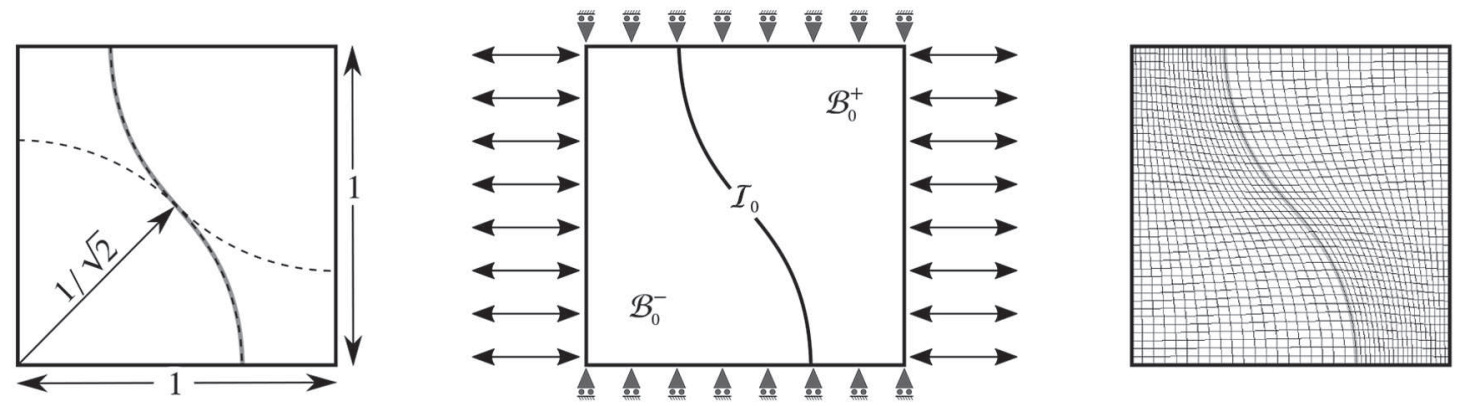

Figure 4. Unit square with interface: geometry (left), boundary conditions (center), and discretization (right). The domain is extended via prescribed displacements at the edges. Lateral deformations are prevented in the sense that the width of the domain cannot vary throughout the deformation process.

of the traction jump across an interface without introducing too much complexity. For the two-dimensional problem of interest here, both parameters $\bar{\mu}$ and $\bar{\lambda}$ serve the same purpose and resist against the change of the length of the interface. Therefore, the interface parameter $\bar{\lambda}$ is set to zero and $\bar{\mu}$ remains as the only parameter to capture the elastic resistance along the interface. The orthogonal resistance of the interface is captured by the cohesive coefficient $\bar{\alpha}$. The bulk materials on both sides of the interface are assumed to be identical with the material parameters $\mu=10\left[\mathrm{~N} / \mathrm{mm}^{2}\right]$ and $\lambda=40\left[\mathrm{~N} / \mathrm{mm}^{2}\right]$ associated with the free energy density (39). The interface material parameters vary among different examples and, for each example, are given within the corresponding section. Obviously, the choice of material parameters depend on the specific application of interest. Nevertheless, the main observations here hold qualitatively, in general.

\section{I. Cohesive interface model}

The first set of examples deal with the cohesive interface model. The significance of this model for the current study is that the cohesive interface model relies on the traction continuity condition. As will be illustrated shortly, the traction continuity condition holds only in the material configuration, but not in the spatial configuration. More precisely, the Piola traction field remains continuous across the interface while the Cauchy traction field suffers a jump. Furthermore, it is shown that for the example of interest here, the traction jump in the spatial configuration increases when increasing the prescribed deformation but not proportionally. In other words, while the traction continuity condition does not hold in the spatial configuration, the relation between the traction jump and the deformation can be very complex and unknown, in general. For this example, the interface cohesive coefficient $\bar{\alpha}=100\left[\mathrm{~N} / \mathrm{mm}^{3}\right]$ and the interface elastic resistance along the interface is excluded entirely.

Figure 5 illustrates the stress distribution on the domain due to the prescribed displacement on the edges. The lines correspond to the $x x$-component of the stress along the upper edge of the specimen. The solid line corresponds to the $x x$-component of the Piola stress and the dashed line corresponds to the $x x$-component of the Cauchy stress. On the upper edge, the interface normal does not throughout the deformation. The interface normal on the upper edge coincides with the unit basis vector along the $x$-direction and, therefore, the stress jump in the $x x$-direction corresponds exactly to the traction jump in the same configuration. More precisely, on the upper edge of the specimen $\llbracket \boldsymbol{t}_{0} \rrbracket_{x}=\llbracket \boldsymbol{P} \rrbracket_{x x}$ and $\llbracket \boldsymbol{t}_{t} \rrbracket_{x}=\llbracket \boldsymbol{\sigma} \rrbracket_{x x}$. This property on the upper edge allows us to interpret the stress jump across the interface in Figure 5 as the traction jump without further implications. Different pairs of lines correspond to different stretches prescribed on the domain. For instance, 20\% means that the unit-length domain is stretched by $20 \%$. It is clear that the Piola traction jump vanishes regardless of the prescribed stretch. In contrast, the Cauchy traction jump exists due to finite deformations. As expected, the Cauchy traction jump vanishes at the reference configuration as both the Piola and Cauchy stresses coincide. Increasing the prescribed deformation on the edges of the domain leads to a more pronounced stress jump at the interface. Figure 6 demonstrates the Cauchy traction jump at the interface versus the prescribed deformation. The Cauchy traction jump corresponds to the $x$-component of the traction on the upper edge of the specimen. For various stretches, the stress distribution on the domain is illustrated. It can be clearly observed that at $1 \%$ stretch, the stress jump at the interface is rather negligible. In contrast, the stress distribution at $90 \%$ stretch shows a noticeable jump at the interface. The numerical results illustrated in Figures 5 and 6 clarify the issue 


\section{O H E S I V E I N T E R F A C E M O D E L}


material configuration

Piola stress distribution

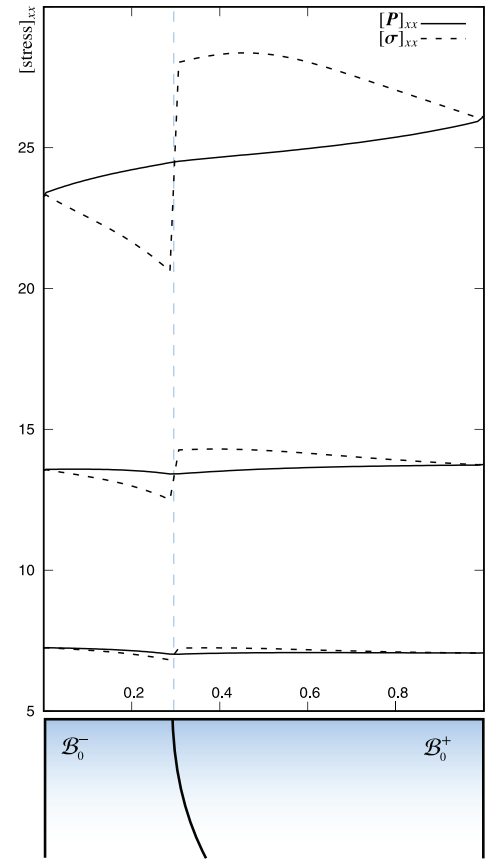

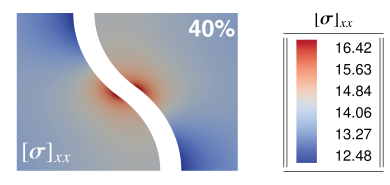

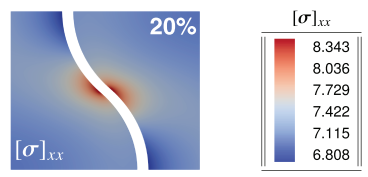

spatial configuration

Cauchy stress distribution

Figure 5. Stress distribution on the domain due to the prescribed displacement on the edges for the cohesive interface model. The lines correspond to the $x x$-component of the stress along the upper edge of the specimen. The solid line corresponds to the $x x$-component of the Piola stress and the dashed line corresponds to the $x x$-component of the Cauchy stress. Different pairs of lines correspond to different stretches prescribed on the domain. It is clear that the Piola traction jump vanishes regardless of the prescribed stretch. In contrast, the Cauchy traction jump exists due to finite deformations. For the cohesive interface model, the displacement jump across the interface does not vanish.

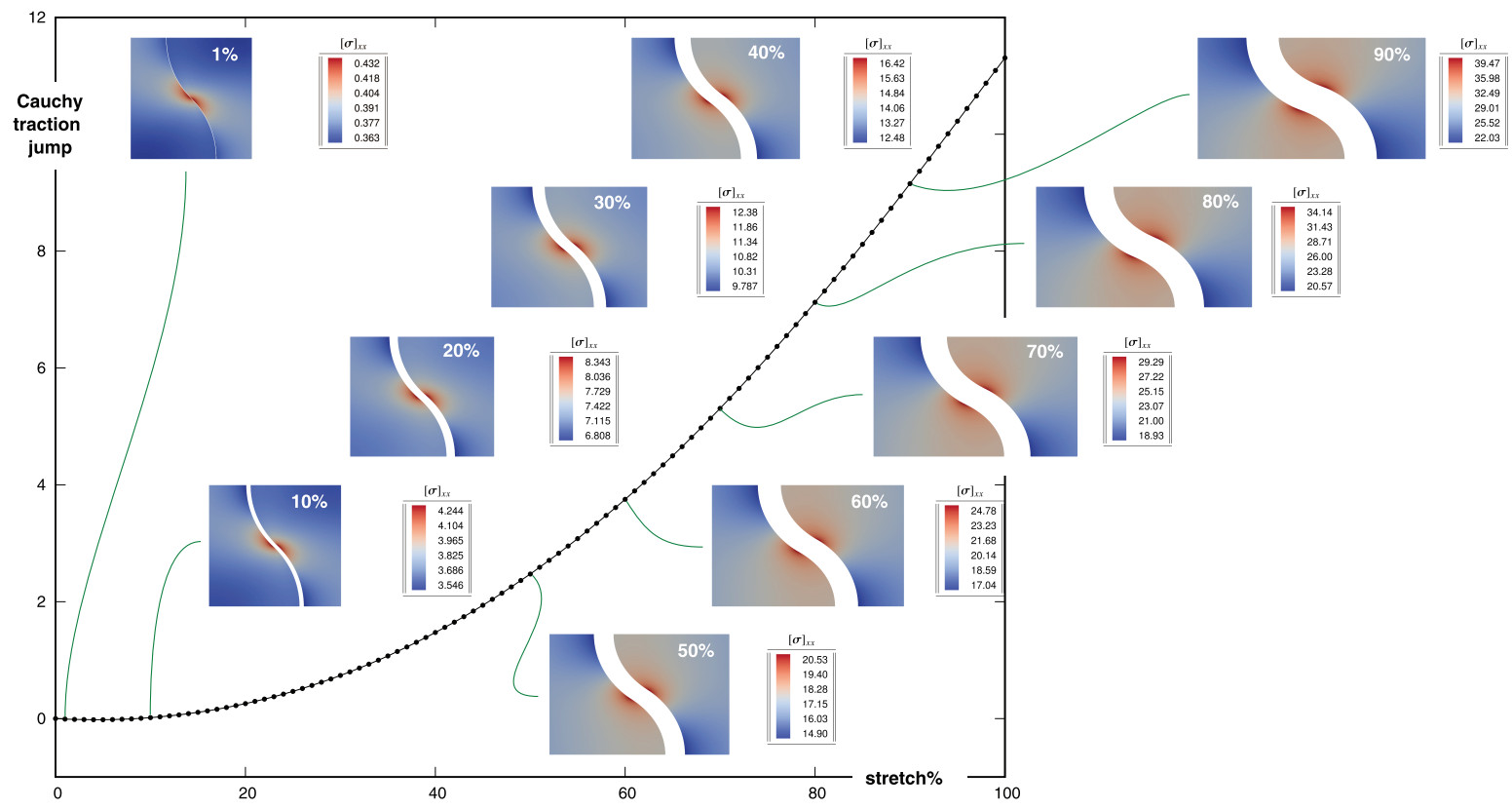

Figure 6. Cauchy traction jump versus prescribed displacement on the edges of the domain for the cohesive interface model. The Cauchy traction jump corresponds to the $x$-component of the traction on the upper edge of the specimen. Obviously, increasing the prescribed deformation on the edges of the domain leads to a more pronounced stress jump at the interface. 


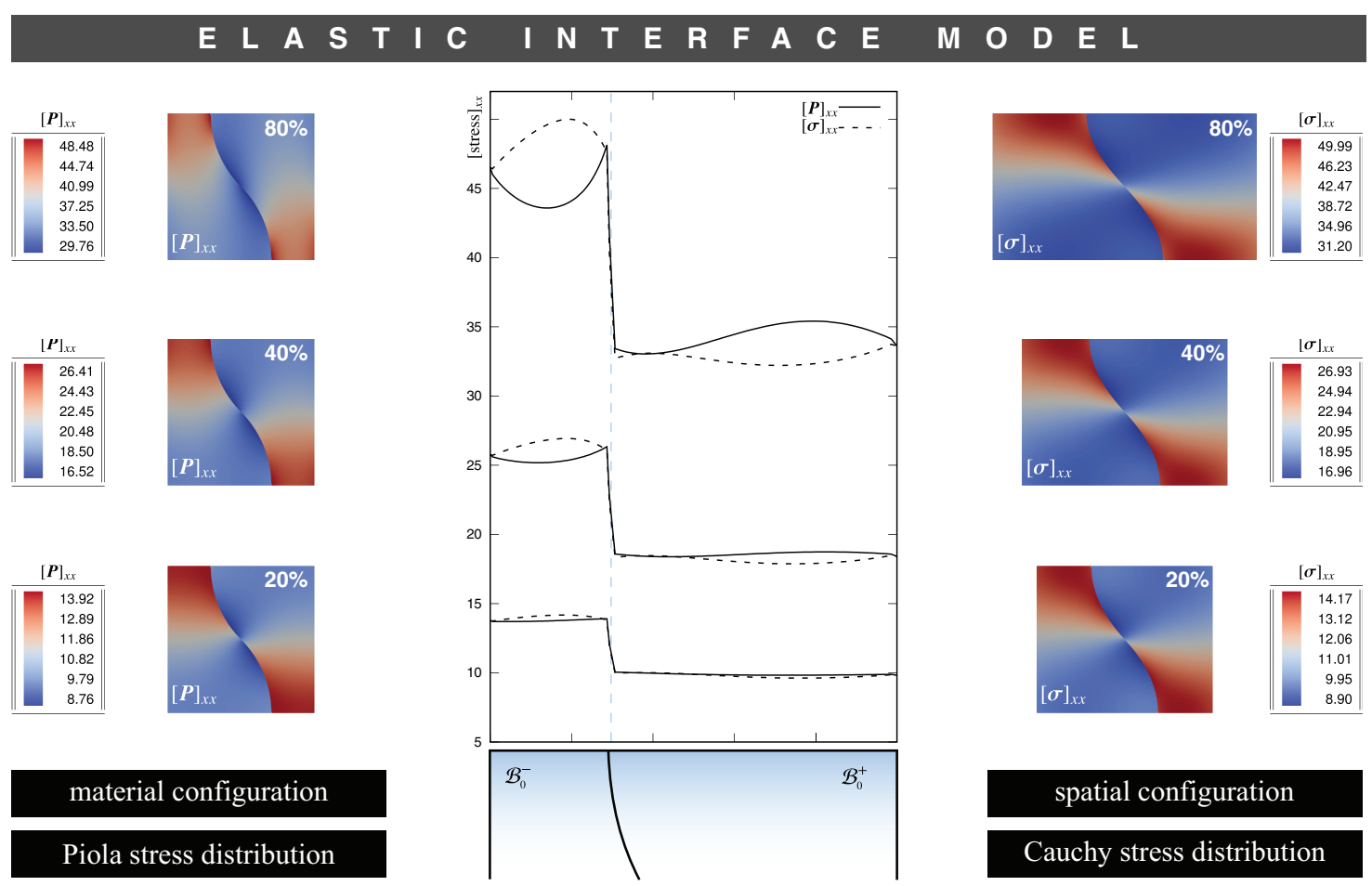

Figure 7. Stress distribution on the domain due to the prescribed displacement on the edges for the elastic interface model. The lines correspond to the $x x$-component of the stress along the upper edge of the specimen. The solid line corresponds to the $x x$-component of the Piola stress and the dashed line corresponds to the $x x$-component of the Cauchy stress. Different pairs of lines correspond to different stretches prescribed on the domain. It is clear that neither the Piola traction jump nor the Cauchy traction jump vanishes regardless of the prescribed stretch. Unlike the cohesive interface mode, for the elastic interface model, the displacement field is continuous across the interface and, thus, the displacement jump across the interface vanishes identically.

of the traction jump at the interface for the cohesive interface model and the significance of the Piola traction jump versus Cauchy traction jump at finite deformations in a geometrically exact theory.

\subsection{Elastic interface model}

The next set of examples illustrates the importance of the elastic interface model on the domain and, in particular, the traction jump across the interface in analogy to the numerical study in Section 3.1. For this example, the interface in-plane elastic parameter $\bar{\mu}=100[\mathrm{~N} / \mathrm{mm}]$ and the interface is assumed to be coherent and thus, $\llbracket \varphi \rrbracket=\mathbf{0}$ across the interface. The geometry and boundary conditions are in accordance with the previous examples and Figure 4. Figure 7 gathers the stress distribution on the domain due to the prescribed displacement on the edges for the elastic interface model. The lines correspond to the $x x$-component of the stress along the upper edge of the specimen. The solid line corresponds to the $x x$-component of the Piola stress and the dashed line corresponds to the $x x$-component of the Cauchy stress. Different pairs of lines correspond to different stretches prescribed on the domain. In contrast to the cohesive interface model, it is clear that neither the Piola traction jump nor the Cauchy traction jump vanishes across the elastic interface model. The stress jump across the interface follows from the generalized Young-Laplace equation as $\llbracket \boldsymbol{t}_{0} \rrbracket=\overline{\operatorname{Div}} \overline{\boldsymbol{P}}$ and $\llbracket \boldsymbol{t}_{t} \rrbracket=\overline{\operatorname{div}} \overline{\boldsymbol{\sigma}}$ in the material and spatial configurations, respectively. Note, the stress profile varies for different prescribed deformations and different stress measures. Furthermore, unlike the cohesive interface model, the displacement field is continuous across the interface and suffers no jump.

\subsection{General interface model}

The numerical examples in Sections 3.1 and 3.2 demonstrate the influence of the cohesive and elastic interface models, respectively. The next set of examples aims to depict the influence of the general interface model. The 


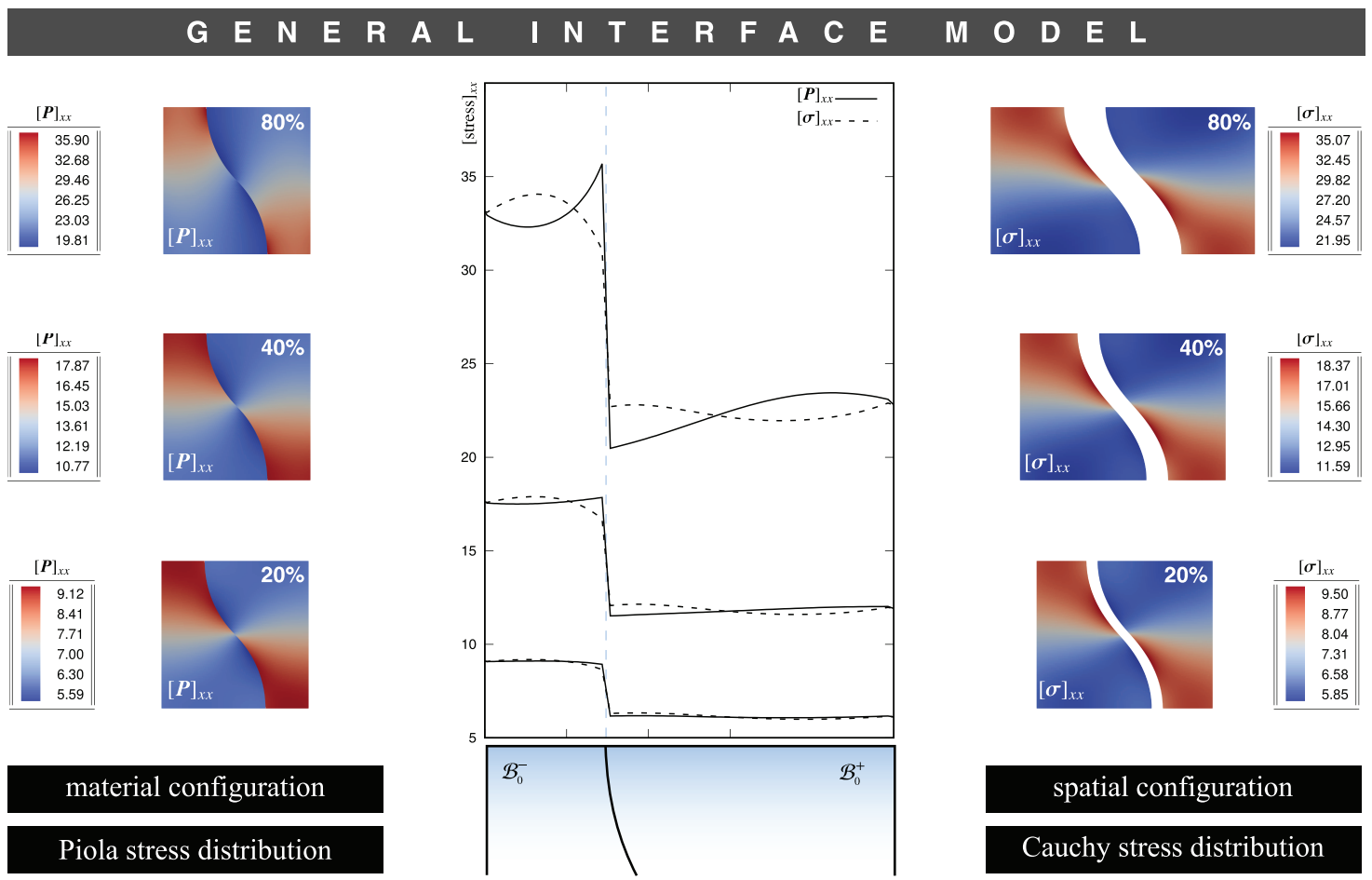

Figure 8. Stress distribution on the domain due to the prescribed displacement on the edges for the general interface model. The lines correspond to the $x x$-component of the stress along the upper edge of the specimen. The solid line corresponds to the $x x$ component of the Piola stress and the dashed line corresponds to the $x x$-component of the Cauchy stress. Different pairs of lines correspond to different stretches prescribed on the domain. It is clear that neither the Piola traction jump nor the Cauchy traction jump vanishes regardless of the prescribed stretch. For the general interface model, the displacement field is discontinuous across the interface and, thus, the displacement jump does vanish, in general.

geometry and boundary conditions are similar to the previous examples and Figure 4. For this example, the interface in-plane elastic parameter $\bar{\mu}=100[\mathrm{~N} / \mathrm{mm}]$ and the interface cohesive coefficient $\bar{\alpha}=100\left[\mathrm{~N} / \mathrm{mm}^{3}\right]$. Figure 8, in analogy to Figures 5 and 7, shows the stress distribution on the domain due to the prescribed displacement on the edges.

Similar to the elastic interface model, the traction jump across the interface does not vanish in either configuration. That is, both the Piola traction jump as well as the Cauchy traction jump suffer a jump across the general interface model. This is in contrast to the cohesive interface model for which the Piola traction jump across the interface vanishes. In contrast to the elastic interface model and similar to the cohesive interface model, the displacement field suffers a jump across the interface. Therefore, it is clear that the general interface model bears similarities as well as differences compared with both the cohesive and elastic interface models.

\subsection{Three-dimensional example}

So far, the numerical examples were devised to illustrate the interface behavior and to highlight the similarities and dissimilarities among different interface models. For the sake of clarity, the numerical examples were limited to two dimensions. Nevertheless, the established theory as well as the numerical framework is general and not limited to any particular geometry or problem dimension. For the sake of completeness, a set of threedimensional numerical examples are gathered next in Figure 9.

The examples refer to a volumetric expansion of a unit cube with a spherical inclusion of radius 0.2 at the center of the cube. The interface is between the inclusion and the matrix and behaves according to the general interface model. To better capture the influence of the interface, both the inclusion and the matrix are assumed to have the same material parameters $\mu=10\left[\mathrm{~N} / \mathrm{mm}^{2}\right]$ and $\lambda=40\left[\mathrm{~N} / \mathrm{mm}^{2}\right]$ associated with the free energy density (39). The interface material parameters vary among different rows and for each row, are given on the first column. The cube is deformed according to an expansion of $10 \%$ prescribed on its facets. The 
reference
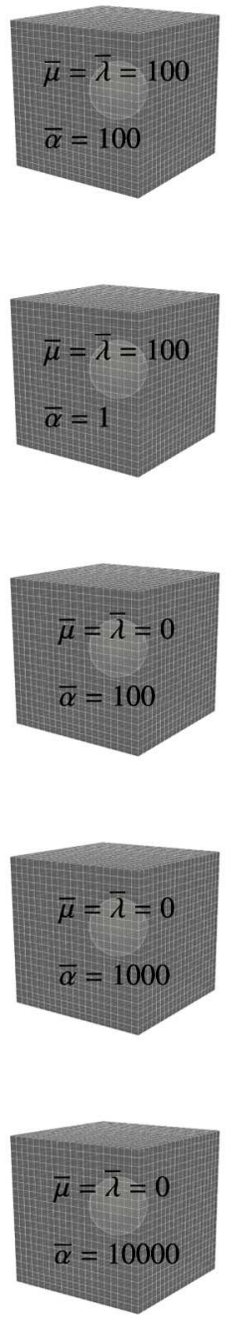

$1 \%$ expansion
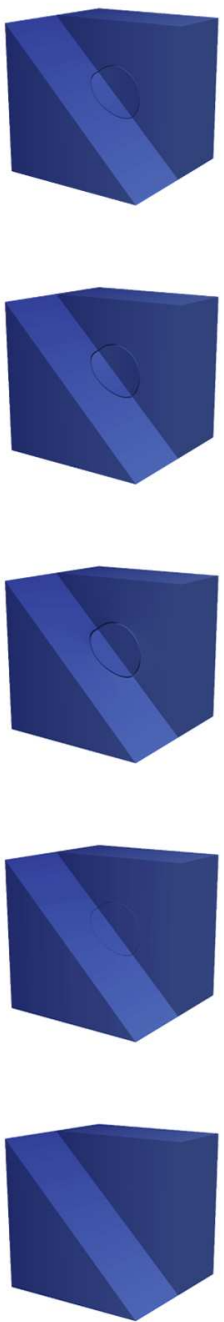

$5 \%$ expansion
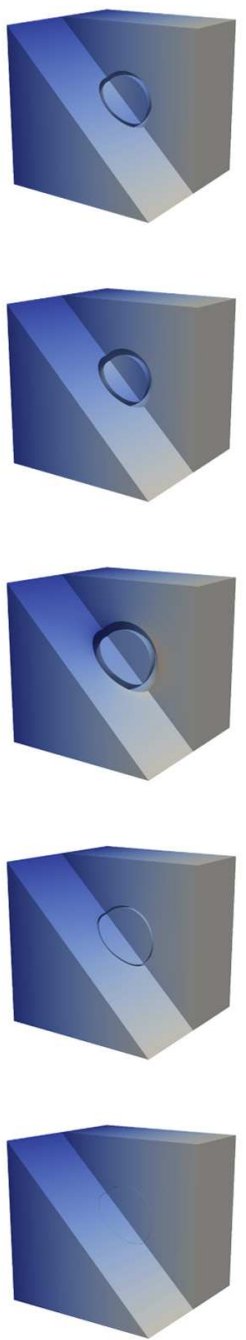

\section{$10 \%$ expansion}
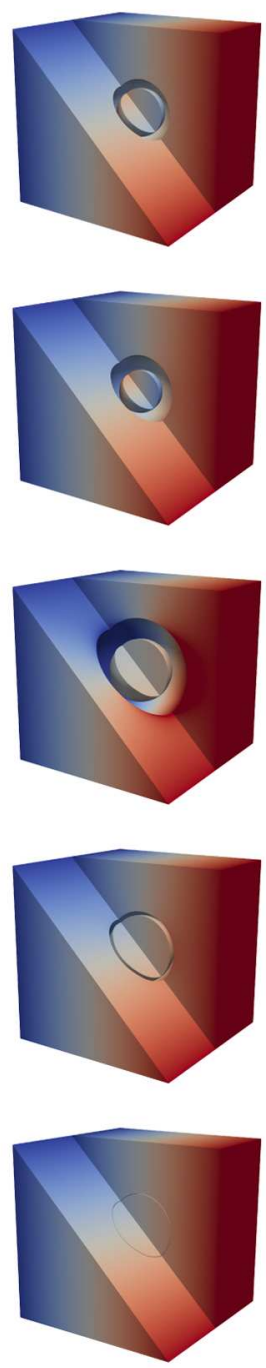

\section{$[u]_{x} \mathbf{0 . 0 0}$}

0.05

0.10

Figure 9. Distribution of the displacement on the domain in the $x$-direction due to the prescribed expansion on the faces for various interface parameters. Different columns correspond to different prescribed expansions. Different rows correspond to different combinations of interface parameters for the general interface model. The last three rows coincide with the cohesive interface model for different cohesive coefficient $\bar{\alpha}$. The cohesive coefficient $\bar{\alpha}$ indicates the interface resistance against opening and, therefore, the displacement jump almost vanishes in the last row.

displacement field in the cube is illustrated for different expansions on each column. For the general interface model, the displacement field is not continuous across the interface and, therefore, a jump of the displacement is always observed. However, the displacement jump decreases for the increasing cohesive coefficient, as expected. More precisely, the cohesive coefficient $\bar{\alpha}$ indicates the interface resistance against opening and at the limit of $\bar{\alpha} \rightarrow \infty$, the interface shall remain virtually coherent.

In the first row of Figure 9, the interface parameters are assumed to be $\bar{\mu}=\bar{\lambda}=100$ and $\bar{\alpha}=100$ associated with the in-plane and orthogonal behavior of the general interface model, respectively. The displacement jump at the interface and its increase due to increasing expansion can be appreciated. On the second row, the interface in-plane parameters are identical to the first row as $\bar{\mu}=\bar{\lambda}=100$, but the cohesive coefficient $\bar{\alpha}=1$ indicates a very weak resistance against opening at the interface. This can be clearly seen by comparing the first two rows. 
For the last three rows, the interface elastic constants are set to zero meaning that the interface does not possess any in-plane resistance and, thus, the general interface model coincides with a cohesive one. The cohesive interfaces on the last three rows have different resistance against opening. It is obvious that increasing the cohesive coefficient, as expected, reduces the displacement jump across the interface. Eventually, the displacement jump across the interface almost vanishes for a relatively large $\bar{\alpha}$ on the last row.

\section{Concluding remarks}

Commonly accepted strategies to capture interface behavior fall into two categories of elastic or cohesive interface models. It is relatively well-established that the traction field suffers a jump across the elastic interface model while it remains continuous for the cohesive interface model. The notion of "traction continuity" across an interface at finite deformations has been elaborated on in this article. In doing so, the general interface model has been rigorously formulated in both the material and spatial configurations and the traction jump across the interface for each configuration has been highlighted. The general interface model is particularly interesting since it reduces to both the elastic and cohesive interface models at its limit cases and can fill the gap between the two extreme interface models. It has clearly been shown that the elastic interface model suffers a traction jump in both the material and spatial configurations according to a generalized Young-Laplace equation. For the cohesive interface model, however, while the traction field is continuous in the material configuration, it can suffer a jump in the spatial configuration which is particularly relevant to finite deformations. The theoretical findings for various interface models have been carefully illustrated via a series of two-dimensional and threedimensional numerical examples using the finite element method. Typical applications of the interface models include nano-materials due to the increasing area-to-volume ratio at smaller dimensions.

\section{Funding}

The author received no financial support for the research, authorship, and/or publication of this article.

\section{ORCID iD}

Ali Javili (iD http://orcid.org/0000-0001-7965-7088

\section{References}

[1] Matous, K, Kulkarni, MG, and Geubelle, PH. Multiscale cohesive failure modeling of heterogeneous adhesives. $J$ Mech Phys Solids 2008; 56(4): 1511-1533.

[2] McBride, A, Mergheim, J, Javili, A, et al. Micro-to-macro transitions for heterogeneous material layers accounting for in-plane stretch. J Mech Phys Solids 2012; 60(6): 1221-1239.

[3] Fried, E, and Grach, G. An order-parameter-based theory as a regularization of a sharp-interface theory for solid--solid phase transitions. Arch Rat Mech Anal 1997; 138(4): 355-404.

[4] Petryk, H, and Stupkiewicz, S. Interfacial energy and dissipation in martensitic phase transformations. Part I: Theory. $J$ Mech Phys Solids 2010; 58(3): 390-408.

[5] Petryk, H, Stupkiewicz, S, and Maciejewski, G. Interfacial energy and dissipation in martensitic phase transformations. Part II: Size effects in pseudoelasticity. J Mech Phys Solids 2010; 58(3): 373-389.

[6] Levitas, VI, and Warren, JA. Phase field approach with anisotropic interface energy and interface stresses: Large strain formulation. J Mech Phys Solids 2016; 91: 94-125.

[7] Tuma, K, Stupkiewicz, S, and Petryk, H. Size effects in martensitic microstructures: Finite-strain phase field model versus sharp-interface approach. J Mech Phys Solids 2016; 95: 284-307.

[8] Bövik, P. On the modelling of thin interface layers in elastic and acoustic scattering problems. Q J Mech Appl Math 1994; 47(1): $17-42$.

[9] Benveniste, Y, and Miloh, T. Imperfect soft and stiff interfaces in two-dimensional elasticity. Mech Mater 2001; 33(6): 309-323.

[10] Benveniste, Y. A general interface model for a three-dimensional curved thin anisotropic interphase between two anisotropic media. J Mech Phys Solids 2006; 54(4): 708-734.

[11] Monchiet, V, and Bonnet, G. Interfacial models in viscoplastic composites materials. Int J Eng Sci 2010; 48(12): 1762-1768.

[12] Benveniste, Y, and Milton, GW. The effective medium and the average field approximations vis-a-vis the Hashin-Shtrikman bounds. I. The self-consistent scheme in matrix-based composites. J Mech Phys Solids 2010; 58(7): 1026-1038.

[13] Benveniste, Y, and Milton, GW. The effective medium and the average field approximations vis-a-vis the Hashin-Shtrikman bounds. II. The generalized self-consistent scheme in matrix-based composites. J Mech Phys Solids 2010; 58(7): 1039-1056. 
[14] Gu, ST, Monteiro, E, and He, QC. Coordinate-free derivation and weak formulation of a general imperfect interface model for thermal conduction in composites. Composites Sci Technol 2011; 71(9): 1209-1216.

[15] $\mathrm{Gu}, \mathrm{ST}$, and $\mathrm{He}, \mathrm{QC}$. Interfacial discontinuity relations for coupled multifield phenomena and their application to the modeling of thin interphases as imperfect interfaces. J Mech Phys Solids 2011; 59(7): 1413-1426.

[16] Pavanello, F, Manca, F, Luca Palla, P, et al. Generalized interface models for transport phenomena: Unusual scale effects in composite nanomaterials. J Appl Phys 2012; 112: 1-10.

[17] Gu, ST, Liu, JT, and He, QC. Size-dependent effective elastic moduli of particulate composites with interfacial displacement and traction discontinuities. International Journal of Solids and Structures 2014; 51(13): 2283-2296.

[18] Chen, T, Chiu, MS, and Weng, CN. Derivation of the generalized Young-Laplace equation of curved interfaces in nanoscaled solids. J Appl Phys 2006; 100(7): 074308.

[19] Javili, A, Mcbride, A, and Steinmann, P. Thermomechanics of solids with lower-dimensional energetics: On the importance of surface, interface, and curve structures at the nanoscale. A unifying review. Appl Mech Rev 2013; 65(1): 010802.

[20] Moeckel, GP. Thermodynamics of an interface. Arch Rat Mech Anal 1975; 57(3): 255-280.

[21] Murdoch, AI. A thermodynamical theory of elastic material interfaces. QJ Mech Appl Math 1976; 29(3): $245-275$.

[22] Daher, N, and Maugin, GA. The method of virtual power in continuum mechanics application to media presenting singular surfaces and interfaces. Acta Mechanica 1986; 60(3-4): 217-240.

[23] Dell'Isola, F, and Romano, A. On the derivation of thermomechanical balance equations for continuous systems with a nonmaterial interface. Int J Eng Sci 1987; 25(11-12): 1459-1468.

[24] Fried, E, and Gurtin, ME. Thermomechanics of the interface between a body and its environment. Continuum Mech Thermodyn 2007; 19(5): 253-271.

[25] Javili, A, Ottosen, NS, Ristinmaa, M, et al. Aspects of interface elasticity theory. Math Mech Solids 2017; DOI: $10.1177 / 1081286517699041$.

[26] Gurtin, ME, and Murdoch, AI. A continuum theory of elastic material surfaces. Arch Rat Mech Anal 1975; 57(4): $291-323$.

[27] Gurtin, ME, Weissmüller, J, and Larche, F. A general theory of curved deformable interfaces in solids at equilibrium. Philos Mag A 1998; 78(5): 1093-1109.

[28] Steigmann, DJ, and Ogden, RW. Elastic surface-substrate interactions. Proc R Soc A Math Phys Eng Sci 1999; 455(1982): 437-474.

[29] Fried, E, and Todres, RE. Mind the gap: The shape of the free surface of a rubber-like material in proximity to a rigid contactor. J Elasticity 2005; 80(1-3): 97-151.

[30] Huang, ZP, and Wang, J. A theory of hyperelasticity of multi-phase media with surface/interface energy effect. Acta Mechanica 2006; 182(3-4): 195-210.

[31] Steinmann, P. On boundary potential energies in deformational and configurational mechanics. J Mech Phys Solids 2008; 56(3): 772-800.

[32] Dingreville, R, and Qu, J. Interfacial excess energy, excess stress and excess strain in elastic solids: Planar interfaces. $J$ Mech Phys Solids 2008; 56(5): 1944-1954.

[33] Duan, HL, Wang, J, and Karihaloo, BL. Theory of Elasticity at the Nanoscale. Adv Appl Mech 2009; 42: 1-68.

[34] Wang, Y, Weissmüller, J, and Duan, HL. Mechanics of corrugated surfaces. J Mech Phys Solids 2010; 58(10): 1552-1566.

[35] Wang, ZQ, Zhao, YP, and Huang, ZP. The effects of surface tension on the elastic properties of nano structures. Int J Eng Sci 2010; 48(2): 140-150.

[36] Altenbach, H, and Eremeyev, VA. On the shell theory on the nanoscale with surface stresses. Int J Eng Sci 2011; 49(12): 12941301.

[37] Chhapadia, P, Mohammadi, P, and Sharma, P. Curvature-dependent surface energy and implications for nanostructures. $J$ Mech Phys Solids 2011; 59(10): 2103-2115.

[38] Zemlyanova, AY. The effect of a curvature-dependent surface tension on the singularities at the tips of a straight interface crack. QJ Mech Appl Math 2013; 66(2): 199-219.

[39] Dingreville, R, Hallil, A, and Berbenni, S. From coherent to incoherent mismatched interfaces: A generalized continuum formulation of surface stresses. J Mech Phys Solids 2014; 72(1): 40-60.

[40] Gao, X, Huang, Z, Qu, J, et al. A curvature-dependent interfacial energy-based interface stress theory and its applications to nano-structured materials: (I) General theory. J Mech Phys Solids 2014; 66(1): 59-77.

[41] Cordero, NM, Forest, S, and Busso, EP. Second strain gradient elasticity of nano-objects. J Mech Phys Solids 2016; $97: 92-124$.

[42] Liu, L, Yu, M, Lin, H, et al. Deformation and relaxation of an incompressible viscoelastic body with surface viscoelasticity. $J$ Mech Phys Solids 2017; 98: 309-329.

[43] Barenblatt, GI. The formation of equilibrium cracks during brittle fracture. General ideas and hypotheses. Axially-symmetric cracks. J Appl Math Mech 1959; 23(3): 622-636.

[44] Barenblatt, GI. The mathematical theory of equilibrium cracks in brittle fracture. Adv Appl Mech 1962; 7: 55-129.

[45] Dugdale, D. Yielding of steel sheets containing slits. J Mech Phys Solids 1960; 8(2): 100-104.

[46] Needleman, A. A continuum model for void nucleation by inclusion debonding. J Appl Mech 1987; 54: 525-531.

[47] $\mathrm{Xu}, \mathrm{XP}$, and Needleman, A. Numerical simulations of fast crack growth in brittle solids. J Mech Phys Solids 1994; 42(9): 1397-1434. 
[48] Ortiz, M, and Pandolfi, A. Finite-deformation irreversible cohesive elements for three-dimensional crack-propagation analysis. Int J Numer Meth Eng 1999; 44(9): 1267-1282.

[49] Tijssens, MG, Sluys, BL, and Van der Giessen, E. Numerical simulation of quasi-brittle fracture using damaging cohesive surfaces. Eur J Mech A Solids 2000; 19(5): 761-779.

[50] Alfano, G, and Crisfield, MA. Finite element interface models for the delamination analysis of laminated composites: mechanical and computational issues. Int J Numer Meth Eng 2001; 50: 1701-1736.

[51] Gasser TC, and Holzapfel GA. Geometrically non-linear and consistently linearized embedded strong discontinuity models for 3D problems with an application to the dissection analysis of soft biological tissues. Comput Meth Appl Mech Eng 2003; 192(47-48): 5059-5098.

[52] van den Bosch, MJ, Schreurs, PJG, and Geers, MGD. An improved description of the exponential Xu and Needleman cohesive zone law for mixed-mode decohesion. Eng Fract Mech 2006; 73(9): 1220-1234.

[53] Fagerström, M and Larsson, R. Theory and numerics for finite deformation fracture modelling using strong discontinuities. Int $J$ Numer Meth Eng 2006; 66(6): 911-948.

[54] Charlotte, M, Laverne, J, and Marigo, JJ. Initiation of cracks with cohesive force models: a variational approach. Eur J Mech A Solids 2006; 25(4): 649-669.

[55] Park, K, Paulino, GH, and Roesler, JR. A unified potential-based cohesive model of mixed-mode fracture. J Mech Phys Solids 2009; 57(6): 891-908.

[56] Park, K, and Paulino, GH. Cohesive zone models: a critical review of traction-separation relationships across fracture surfaces. Appl Mech Rev 2013; 64(6): 060802.

[57] Dimitri, R, Trullo, M, De Lorenzis, L, et al. Coupled cohesive zone models for mixed-mode fracture: A comparative study. Eng Fract Mech 2015; 148: 145-179.

[58] Wu, C, Gowrishankar, S, Huang, R, et al. On determining mixed-mode traction-separation relations for interfaces. Int J Fract 2016; 202(1): 1-19.

[59] Qian, J, Lin, J, Xu, GK, et al. Thermally assisted peeling of an elastic strip in adhesion with a substrate via molecular bonds. $J$ Mech Phys Solids 2017; 101: 197-208.

[60] Marsden, JE, and Hughes, TJR. Mathematical Foundations of Elasticity. New York: Dover, 1994.

[61] Holzapfel, GA. Nonlinear Solid Mechanics: A Continuum Approach for Engineering. Hoboken, NJ: John Wiley \& Sons, 2000.

[62] Gurtin, ME, Fried, E, and Anand, L. The Mechanics and Thermodynamics of Continua. Cambridge: Cambridge University Press, 2009.

[63] Bowen, RM, and Wang, CC. Introduction to Vectors and Tensors: Linear and Multilinear Algebra. New York: Plenum Press, 1976.

[64] Kreyszig, E. Differential Geometry. New York: Dover, 1991.

[65] Ciarlet, PG. An Introduction to Differential Geometry with Applications to Elasticity. New York: Springer, 2005.

[66] Steinmann, P. Geometrical Foundations of Continuum Mechanics: An Application to First-and Second-Order Elasticity and Elasto-Plasticity. New York: Springer, 2015.

[67] Javili, A. Variational formulation of generalized interfaces for finite deformation elasticity. Math Mech Solids 2017; DOI: $10.1177 / 1081286517719938$.

[68] Javili, A, Steinmann, P, and Mosler, J. Micro-to-macro transition accounting for general imperfect interfaces. Comput Meth Appl Mech Eng 2017; 317: 274-317.

[69] Javili, A, Dell'Isola, F, and Steinmann, P. Geometrically nonlinear higher-gradient elasticity with energetic boundaries. $J$ Mech Phys Solids 2013; 61(12): 2381-2401.

[70] Vossen, BG, Schreurs, PJG, van der Sluis, O, et al. On the lack of rotational equilibrium in cohesive zone elements. Comput Meth Appl Mech Eng 2013; 254: 146-153.

[71] Ogden, R. Large deformation isotropic elasticity - on the correlation of theory and experiment for incompressible rubberlike solids. Proc R Soc A 1972; 326(1567): 565-584.

[72] Linder, C, Tkachuk, M, and Miehe, C. A micromechanically motivated diffusion-based transient network model and its incorporation into finite rubber viscoelasticity. J Mech Phys Solids 2011; 59(10): 2134-2156. 